\title{
Invariance of quantum rings under ordinary flops I: Quantum corrections and reduction to local models
}

\author{
Yuan-Pin Lee, Hui-Wen Lin and Chin-Lung Wang
}

\begin{abstract}
This is the first of a sequence of papers proving the quantum invariance under ordinary flops over an arbitrary smooth base. In this first part, we determine the defect of the cup product under the canonical correspondence and show that it is corrected by the small quantum product attached to the extremal ray. We then perform various reductions to reduce the problem to local models. In part II ("Invariance of quantum rings under ordinary flops II", Algebraic Geometry, 2016), we develop a quantum Leray-Hirsch theorem and use it to show that the big quantum cohomology ring is invariant under analytic continuation in the Kähler moduli space for ordinary flops of split type. In part III (Lee, Lin, Qu and Wang, "Invariance of quantum rings under ordinary flops III", Cambridge Journal of Mathematics, 2016), we remove the splitting condition by developing a quantum splitting principle, and hence solve the problem completely.
\end{abstract}

\section{Introduction}

\subsection{Background review}

Two complex manifolds $X$ and $X^{\prime}$ are $K$-equivalent, denoted by $X={ }_{K} X^{\prime}$, if there are proper birational morphisms $\phi: Y \rightarrow X$ and $\phi^{\prime}: Y \rightarrow X^{\prime}$ such that $\phi^{*} K_{X}=\phi^{*} K_{X^{\prime}}$, where $K_{X}$ is the canonical divisor". Major examples come from birational minimal models in Mori theory and especially from birational Calabi-Yau manifolds in the mathematical study of string theory. $K$ equivalent projective manifolds share the same Betti and Hodge numbers. It has been conjectured that a canonical correspondence $T \in A\left(X \times X^{\prime}\right)$ exists which induces isomorphisms of cohomology groups and preserves the Poincaré pairing. For a survey, see [Wan04].

However, simple examples show that the classical cup product is generally not preserved under the above correspondence, and this leads to new directions of study in higher-dimensional birational geometry. On the other hand, according to the philosophy of the crepant transformation conjecture and string theory, the quantum product should be more natural and display a certain functoriality not available to the cup product among $K$-equivalent manifolds.

Received 30 July 2014, accepted in final form 01 February 2016.

2010 Mathematics Subject Classification 14N35, $14 \mathrm{E} 30$.

Keywords: quantum cohomology, ordinary flops, analytic continuations, degeneration formula, reconstructions. This journal is (C) Foundation Compositio Mathematica 2016. This article is distributed with Open Access under the terms of the Creative Commons Attribution Non-Commercial License, which permits non-commercial reuse, distribution, and reproduction in any medium, provided that the original work is properly cited. For commercial re-use, please contact the Foundation Compositio Mathematica.

Y.-P. Lee is partially supported by the NSF; H.-W. Lin is partially supported by the MOST; C.-L. Wang is partially supported by the MOST and the MOE. We are particularly grateful to Taida Institute of Mathematical Sciences (TIMS) for its steady support which makes this long-term collaborative project possible. 


\section{INVARIANCE OF QUANTUM RINGS I}

Flops are typical examples of $K$-equivalent birational maps:

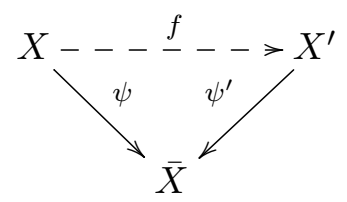

In fact, they form the building blocks for connecting birational minimal models [Kaw08]. The simplest flop is the simple $P^{1}$ flop (Atiyah flop) in dimension 3. It is known that up to deformation, it generates, locally or symplectically, all $K$-equivalent maps for threefolds. The quantum corrections to the cup product by extremal ray invariants in the local 3-dimensional case were first observed by Aspinwall-Morrison and Witten [Wit93] and later globalized by Li-Ruan through the degeneration formula [LR01].

The higher-dimensional generalizations are known as ordinary $P^{r}$ flops (also abbreviated as "ordinary flops" or " $P^{r}$ flops"). The local geometry is encoded in a triple $\left(S, F, F^{\prime}\right)$, where $S$ is a smooth variety and $F$ and $F^{\prime}$ are two rank $r+1$ vector bundles over $S$. If $Z \subset X$ is the $f$-exceptional locus, then there exists a $\bar{\psi}: Z \cong P(F) \rightarrow S \subset \bar{X}$ with fibers spanned by the flopped curves $C \cong P^{1}$ and $N_{Z / X}=\bar{\psi}^{*} F^{\prime} \otimes \mathscr{O}_{Z}(-1)$. A similar structure holds for $Z^{\prime} \subset X^{\prime}$, with $F$ and $F^{\prime}$ exchanged. See Section 2.1 for details. (We note that the Atiyah flop corresponds to $S=$ pt and $r=1$.) Thus it is reasonable to expect that ordinary flops play a vital role in the study of $K$-equivalent maps. For example, up to complex cobordism, any $K$-equivalent map can be decomposed into $P^{1}$ flops [Wan03].

The study of the invariance of quantum products under ordinary flops in higher dimensions was started in [LLW10]. The canonical correspondence is given by the graph closure $\left[\bar{\Gamma}_{f}\right]$, and the quantum invariance under

$$
\mathscr{F}=\left[\bar{\Gamma}_{f}\right]_{*}: \mathrm{QH}(X) \rightarrow \mathrm{QH}\left(X^{\prime}\right)
$$

is proved for all simple $P^{r}$ flops, that is, all $P^{r}$ flops with $S=$ pt. Here the quantum cohomology rings $\mathrm{QH}(X)$ and $\mathrm{QH}\left(X^{\prime}\right)$ are defined below. The crucial idea is to interpret $\mathscr{F}$-invariance in terms of analytic continuations in Gromov-Witten theory.

Let us explain this point in a little more detail. We use [CK99] as our general reference for early developments in Gromov-Witten $(\mathrm{GW})$ invariants. Let $\bar{M}_{g, n}(X, \beta)$ be the moduli space of stable maps from genus $g$ nodal curves with $n$ marked points to $X$, and let $e_{i}: \bar{M}_{g, n}(X, \beta) \rightarrow X$ be the evaluation maps. The Gromov-Witten potential

$$
F_{g}^{X}(t)=\sum_{n, \beta} \frac{q^{\beta}}{n !}\left\langle t^{n}\right\rangle_{g, n, \beta}^{X}=\sum_{n \geqslant 0, \beta \in \mathrm{NE}(X)} \frac{q^{\beta}}{n !} \int_{\left.\left[\bar{M}_{g, n}(X, \beta)\right]\right]^{\operatorname{virt}}} \prod_{i=1}^{n} e_{i}^{*} t
$$

is a formal function in $t \in H(X)$ and Novikov variables $q^{\beta}$, with $\beta$ an element of $\mathrm{NE}(X)$, the Mori cone of effective classes of 1-cycles. Modulo convergence issues, it is a function on the complexified Kähler cone $\mathcal{K}^{\mathbb{C}_{X}}:=H_{\mathbb{R}}^{1,1}+i \mathcal{K}_{X}$ via

$$
\omega \mapsto q^{\beta}=e^{2 \pi i(\beta \cdot \omega)} .
$$

Under the canonical correspondence $\mathscr{F}$, the potentials $F_{g}^{X}$ and $F_{g}^{X^{\prime}}$ share the same variable $t \in H \cong H(X, \mathbb{C}) \cong H\left(X^{\prime}, \mathbb{C}\right)$. However, $\mathscr{F}$ does not identify $\mathrm{NE}(X)$ with $\mathrm{NE}\left(X^{\prime}\right)$. Indeed, for the flopped curve classes $\ell=[C]$ and $\ell^{\prime}=\left[C^{\prime}\right]$, we have

$$
\mathscr{F} \ell=-\ell^{\prime} \notin \mathrm{NE}\left(X^{\prime}\right) \text {. }
$$


By duality this implies $\mathcal{K}^{\mathbb{C}_{X}} \cap \mathcal{K}^{\mathbb{C}_{X^{\prime}}}=\emptyset$ in $H_{\mathbb{C}}^{2}$. Hence $F_{g}^{X}$ and $F_{g}^{X^{\prime}}$ have different domains and comparison can only make sense after analytic continuation over a certain compactification of $\mathcal{K}^{\mathbb{C}_{X}} \cup \mathcal{K}^{\mathbb{C}_{X^{\prime}}} \subset H_{\mathbb{C}}^{2}$. (Thus the naive Kähler moduli space $\mathcal{K}$ is usually regarded as the closure of the union of all $\mathcal{K}^{\mathbb{C}_{X^{\prime}}}$ with $X^{\prime}={ }_{K} X$.) In other words, we set $\mathscr{F} q^{\beta}=q^{\mathscr{F} \beta}$. Then $\mathscr{F} F_{g}^{X}$ cannot be a formal GW potential of $X^{\prime}$.

In this paper, we will focus on genus zero theory, which carries a quantum product structure, or equivalently a Frobenius structure [Man99]. Let $\left\{T_{\mu}\right\}$ be a basis of $H$ and $\left\{T^{\mu}:=\sum g^{\mu \nu} T_{\nu}\right\}$ the dual basis with respect to the Poincaré pairing, where $g_{\mu \nu}=\left(T_{\mu} \cdot T_{\nu}\right)$ and $\left(g^{\mu \nu}\right)=\left(g_{\mu \nu}\right)^{-1}$ is the inverse matrix. Denote by $t=\sum t^{\mu} T_{\mu}$ a general element of $H$. The big quantum ring $(\mathrm{QH}(X), *)$ uses only the genus zero potential with three or more marked points:

$$
T_{\mu} *_{t} T_{\nu}=\sum_{\kappa} \frac{\partial^{3} F_{0}^{X}}{\partial t^{\mu} \partial t^{\nu} \partial t^{\kappa}}(t) T^{\kappa}=\sum_{\kappa, n \geqslant 0, \beta \in \mathrm{NE}(X)} \frac{q^{\beta}}{n !}\left\langle T_{\mu}, T_{\nu}, T_{\kappa}, t^{n}\right\rangle_{0, n+3, \beta}^{X} T^{\kappa} .
$$

The Witten-Dijkgraaf-Verlinde-Verlinde (WDVV) equation guarantees that $*_{t}$ is a family of associative products on $H$ parameterized by $t \in H$. Equivalently, it equips $H$ with a structure of formal Frobenius manifold $H_{X}$ with a family (in $z \in \mathbb{C}^{\times}$) of integrable (or, equivalently, flat) Dubrovin connections

$$
\nabla^{z}=d-z^{-1} \sum_{\mu} d t^{\mu} \otimes T_{\mu} *_{t}
$$

on the tangent bundle $T H=H \times H$.

There is a natural embedding of $\mathcal{K}^{\mathbb{C}_{X}}$ in $H$. With a suitable choice of coordinates we have $q^{\ell}=e^{2 \pi i t_{\ell}}$ with Kähler constraint $\Im t_{\ell}>0$. Since now $\mathscr{F} q^{\ell}=q^{-\ell^{\prime}}$, the pair $\left\{q^{\ell}, q^{\ell^{\prime}}\right\}$ serves as an atlas for $P^{1}$, the compactification of $\mathbb{C} / \mathbb{Z} \cong \mathbb{C}^{\times}$. This gives the formal $H$ an analytic $P^{1}$ direction. In [LLW10], for simple flops, the structural constants $\partial_{\mu \nu \kappa}^{3} F_{0}^{X}(t)$ for the big quantum product are shown to be analytic (in fact algebraic) in $q^{\ell}$. Moreover, $\mathscr{F}$ identifies $H_{X}$ and $H_{X^{\prime}}$ through analytic continuations over this $P^{1}$. Based on this, in [ILLW12] the Frobenius structure is further exploited to deduce analytic continuation from $F_{g}^{X}$ to $F_{g}^{X^{\prime}}$ for all simple flops and for all $g \geqslant 0$.

\subsection{Outline of the contents}

This is the first of a sequence of papers proving the quantum invariance under ordinary flops over a smooth base. In this first part, we determine the defect of the cup product under the canonical correspondence and show that it is corrected by the small quantum product attached to the extremal ray. We then perform various reductions to local models.

In part II [LLW13], we show that the big quantum cohomology ring is invariant under analytic continuation in the Kähler moduli space for flops of split type. In part III [LLQW16], the final part of this series, we remove the splitting condition by developing a quantum splitting principle, hence solve the problem completely.

In particular, this is the first result on the $K$-equivalence (crepant transformation) conjecture where the local structure of the exceptional loci cannot be deformed to any explicit (for example, toric) geometry and the analytic continuation is non-trivial. As far as we know, this is also the first result for which the analytic continuation is established with non-trivial Birkhoff factorizations.

We give an outline of the contents of this paper below.

Conventions. Throughout this paper, we work on the even cohomology $H=H^{\text {even }}$ to avoid complications with signs. In particular, by degree we always mean the Chow degree. Nevertheless, 


\section{INVARIANCE OF QUANTUM RINGS I}

all our discussions and results work for the full cohomology spaces.

1.2.1 Defect of the cup product under the canonical correspondence. Let $\left\{\bar{T}_{i}\right\}$ be a basis of $H(S)$ with dual basis $\left\{\check{\bar{T}}_{i}\right\}$. Let $h=c_{1}\left(\mathscr{O}_{Z}(1)\right)$ and $H_{k}=c_{k}\left(Q_{F}\right)$, where $Q_{F} \rightarrow Z=P(F)$ is the universal quotient bundle. Similarly, we define $h^{\prime}$ and $H_{k}^{\prime}$ on the $X^{\prime}$ side. The $H_{k}$ are of fundamental importance since

$$
\mathscr{F} H_{k}=(-1)^{r-k} H_{k}^{\prime}
$$

and the dual basis of $\left\{\bar{T}_{i} h^{j}\right\}$ in $H(Z)$ is given by $\left\{\check{\bar{T}}_{i} H_{r-j}\right\}$.

Theorem (Theorem 2.8, topological defect). Let $a_{1}, a_{2}, a_{3} \in H(X)$ with $\sum \operatorname{deg} a_{i}=\operatorname{dim} X$. Then

$$
\begin{aligned}
& \left(\mathscr{F} a_{1} \cdot \mathscr{F} a_{2} \cdot \mathscr{F} a_{3}\right)^{X^{\prime}}-\left(a_{1} \cdot a_{2} \cdot a_{3}\right)^{X} \\
& =(-1)^{r} \sum_{i_{*}, j_{*}}\left(a_{1} \cdot \check{\bar{T}}_{i_{1}} H_{r-j_{1}}\right)^{X}\left(a_{2} \cdot \check{\bar{T}}_{i_{2}} H_{r-j_{2}}\right)^{X} \\
& \quad \times\left(a_{3} \cdot \check{\bar{T}}_{i_{3}} H_{r-j_{3}}\right)^{X}\left(s_{j_{1}+j_{2}+j_{3}-(2 r+1)}\left(F+F^{\prime *}\right) \bar{T}_{i_{1}} \bar{T}_{i_{2}} \bar{T}_{i_{3}}\right)^{S},
\end{aligned}
$$

where $s_{i}$ is the ith Segre class.

1.2.2 Quantum corrections attached to flopping extremal rays. We proceed to calculate the quantum corrections attached to the flopping extremal ray $\mathbb{N} \ell$. Using the calculation, we demonstrate that the "quantum corrected product", combining the classical product and the quantum deformation attached to the extremal ray, is $\mathscr{F}$-invariant after analytic continuation.

The stable map moduli space for the extremal ray has a bundle structure over $S$ :

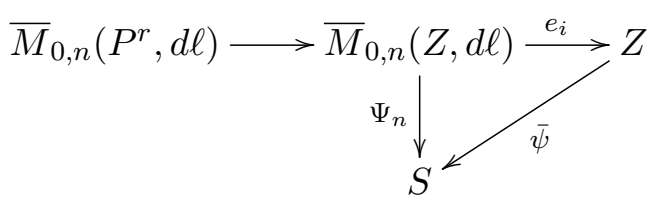

In this case, the GW invariants on $X$ are reduced to twisted invariants on $Z$ by certain obstruction bundles. We define the fiber integral (see Section 3.1 for the details of the notation)

$$
\left\langle\prod_{i=1}^{n} h^{j_{i}}\right\rangle_{d}^{/ S}:=\Psi_{n *}\left(\prod_{i=1}^{n} e_{i}^{*} h^{j_{i}} \cdot e\left(R^{1} f t_{*} e_{n+1}^{*} N_{Z / X}\right)\right) \in A^{\nu}(S)
$$

as a $\bar{\psi}$-relative invariant over $S$, a cycle of codimension $\nu:=\sum j_{i}-(2 r+1+n-3)$. The absolute invariant is obtained by the pairing on $S$ : for $\bar{t}_{i} \in H(S)$,

$$
\left\langle\bar{t}_{1} h^{j_{1}}, \ldots, \bar{t}_{n} h^{j_{n}}\right\rangle_{d}^{X}=\left(\left\langle h^{j_{1}}, \ldots, h^{j_{n}}\right\rangle_{d}^{/ S} \cdot \prod_{i=1}^{n} \bar{t}_{i}\right)^{S}
$$

If $\nu=0$, the invariant reduces to the simple case. This happens for $n=2$, since then $j_{1}=j_{2}=r$. Thus we may calculate extremal functions based on the 2-point case by (divisorial) reconstruction. To state the result, let

$$
\mathbf{f}(q):=\frac{q}{1-(-1)^{r+1} q},
$$

which satisfies the functional equation $\mathbf{f}(q)+\mathbf{f}\left(q^{-1}\right)=(-1)^{r}$. 
For 3-point functions, we show that $W_{\nu}:=\sum_{d \in \mathbb{N}}\left\langle h^{j_{1}}, h^{j_{2}}, h^{j_{3}}\right\rangle_{d}^{/ S} q^{d}$ with $1 \leqslant j_{i} \leqslant r$ lies in $A^{\nu}(S)[\mathbf{f}]$ and is independent of the choices of the $j_{i}$.

Theorem (Theorem 3.6, quantum corrections). The function $W_{\nu}$ is the action on $\mathbf{f}$ by a polynomial in the operator $\delta=q d / d q$ with Chern classes as coefficients. (See Proposition 3.5.) It satisfies

$$
W_{\nu}-(-1)^{\nu+1} W_{\nu}^{\prime}=(-1)^{r} s_{\nu}\left(F+F^{* *}\right) .
$$

This implies that the topological defect is corrected by the 3-point extremal functions. The analytic continuation for $n \geqslant 4$ points follows by reconstruction.

1.2.3 Degeneration analysis. The next step is to prove that the big quantum ring, involving all curve classes, is $\mathscr{F}$-invariant. As a first step, this statement is reduced to a corresponding one on $f$-special descendent invariants on the projective local models

$$
X_{l o c}:=\tilde{E}=P\left(N_{Z / X} \oplus \mathscr{O}\right) \stackrel{p}{\rightarrow} Z
$$

and

$$
X_{l o c}^{\prime}:=\tilde{E}^{\prime}=P\left(N_{Z^{\prime} / X^{\prime}} \oplus \mathscr{O}\right) \stackrel{p^{\prime}}{\rightarrow} Z^{\prime}
$$

by a degeneration analysis.

To compare GW invariants of non-extremal classes, application of the degeneration formula and deformation to the normal cone is well suited for ordinary flops with base $S$. This reduces the problem to local models with induced flop $f: \tilde{E} \rightarrow-\tilde{E}^{\prime}$. The reduction has two steps. The first reduces the problem to relative local invariants $\langle A \mid \varepsilon, \mu\rangle^{(\tilde{E}, E)}$, where $E \subset \tilde{E}$ is the infinity divisor. The second is a further reduction back to absolute local invariants, with possibly descendent insertions coupled to $E$, called of $f$-special type.

The local model $\bar{p}:=\bar{\psi} \circ p: \tilde{E} \rightarrow S$ and the flop $f$ are defined over $S$, with the simple case as fibers. In particular, the kernel of $\bar{p}_{*}: N_{1}(\tilde{E}) \rightarrow N_{1}(S)$ is spanned by the $p$-fiber line class $\gamma$ and $\bar{\psi}$-fiber line class $\ell$. The correspondence $\mathscr{F}$ is compatible with $\bar{p}$. Namely,

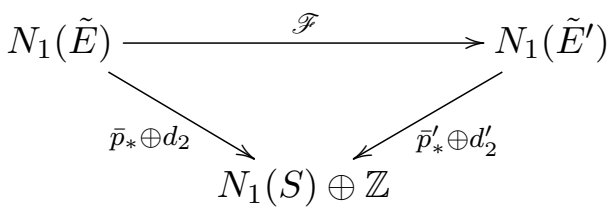

is commutative. Here we write a class $\beta$ in $N_{1}(\tilde{E})$ as $\beta_{S}+d \ell+d_{2} \gamma$ for some $\beta_{S}$ in $N_{1}(S)$ and $d, d_{2} \in \mathbb{Z}$. Thus the functional equation of a generating series $\langle A\rangle$ is equivalent to the functional equations of its various subseries (fiber series) $\langle A\rangle_{\beta_{S}, d_{2}}$ labeled by $\operatorname{NE}(S) \oplus \mathbb{Z}$.

TheOrem 1.1 (Degeneration reduction). To prove $\mathscr{F}\langle\alpha\rangle_{g}^{X} \cong\langle\mathscr{F} \alpha\rangle_{g}^{X^{\prime}}$ for all $\alpha \in H(X)^{\oplus n}$ and $g \leqslant g_{0}$, it is enough to prove the local case $f: \tilde{E} \rightarrow \tilde{E}^{\prime}$ for descendent invariants of $f$-special type:

$$
\mathscr{F}\left\langle A, \tau_{k_{1}} \varepsilon_{1}, \ldots, \tau_{k_{\rho}} \varepsilon_{\rho}\right\rangle_{g, \beta_{S}, d_{2}}^{\tilde{E}} \cong\left\langle\mathscr{F} A, \tau_{k_{1}} \varepsilon_{1}, \ldots, \tau_{k_{\rho}} \varepsilon_{\rho}\right\rangle_{g, \beta_{S}, d_{2}}^{\tilde{E}^{\prime}}
$$

for any $A \in H(\tilde{E})^{\oplus n}, k_{j} \in \mathbb{N} \cup\{0\}, \varepsilon_{j} \in H(E) \subset H(\tilde{E}), g \leqslant g_{0}, \beta_{S} \in \mathrm{NE}(S)$ and $d_{2} \geqslant 0$.

1.2.4 Further reduction to the big quantum ring, quasi-linearity on local models. While the degeneration reduction works for higher genera, for $g=0$ more can be said. Using the topological recursion relation (TRR) and the divisor axiom (for descendent invariants), the $\mathscr{F}$-invariance 


\section{INVARIANCE OF QUANTUM RINGS I}

for $f$-special invariants can be completely reduced to the $\mathscr{F}$-invariance of big quantum rings for local models (Theorem 5.2).

We then employ the divisorial reconstruction [LP04] and the WDVV equation to make a further reduction to an $\mathscr{F}$-invariance statement about elementary $f$-special invariants with at most one special insertion.

To state the result, we now assume $X=X_{\text {loc }}=\tilde{E}$. Since $X \rightarrow S$ is a double projective bundle, $H(X)$ is generated by $H(S)$ and the relative hyperplane classes $h$ for $Z \rightarrow S$ and $\xi$ for $X \rightarrow Z$. This leads to another useful reduction: by moving all the classes $h, \xi$ and $\psi$ into the last insertion (divisorial reconstruction), the problem is reduced to the case

$$
\left\langle\bar{t}_{1}, \ldots, \bar{t}_{n-1}, \bar{t}_{n} \tau_{k} h^{j} \xi^{i}\right\rangle_{\beta_{S}, d_{2}}^{X}
$$

with $\bar{t}_{l} \in H(S)$ and $d_{2} \in \mathbb{Z}$, where $k \neq 0$ only if $i \neq 0$.

By a further application of WDVV equations, the $\mathscr{F}$-invariance can always be reduced to the case $i \neq 0$ even if $k=0$. Since $\xi$ is the class of the infinity divisor, which is within the isomorphism locus of the flop, such an $\mathscr{F}$-invariance statement is intuitively plausible. We call it the type I quasi-linearity property (cf. Theorem 5.5).

The above steps furnish a complete reduction to projective local models $X_{\text {loc }}$, which works for any $F$ and $F^{\prime}$.

To proceed, notice that these descendent invariants are encoded by their generating function, that is, the so-called (big) $J$ function: for $\tau \in H(X)$,

$$
J^{X}\left(\tau, z^{-1}\right):=1+\frac{\tau}{z}+\sum_{\beta, n, \mu} \frac{q^{\beta}}{n !} T_{\mu}\left\langle\frac{T^{\mu}}{z(z-\psi)}, \tau, \ldots, \tau\right\rangle_{0, n+1, \beta}^{X} .
$$

The determination of $J$ usually relies on the existence of $\mathbb{C}^{\times}$-action. Certain localization data $I_{\beta}$ coming from the stable map moduli are of hypergeometric type. For "good" cases, say $c_{1}(X)$ is semipositive and $H(X)$ is generated by $H^{2}$, the sum $I(t)=\sum I_{\beta} q^{\beta}$ determines $J(\tau)$ on the small parameter space $H^{0} \oplus H^{2}$ through the "classical" mirror transform $\tau=\tau(t)$. For a simple flop, $X=X_{\text {loc }}$ is indeed semi-Fano toric and the classical mirror theorem (of Lian-Liu-Yau and Givental) is sufficient [LLW10]. (It turns out that $\tau=t$ and $I=J$ on $H^{0} \oplus H^{2}$.)

For a general base $S$ with given $\mathrm{QH}(S)$, the determination of $\mathrm{QH}(P)$ for a projective bundle $P \rightarrow S$ is far more involved. To allow fiberwise localization to determine the structure of the GW invariants of $X_{\mathrm{loc}}$, the bundles $F$ and $F^{\prime}$ are then assumed to be split bundles. This is the main subject to be studied in part II of this series [LLW13].

Remark 1.2. Results in this paper have been announced by the authors, in increasing degree of generality, at various conferences during 2008-2010; see, for example, [Lin10, Wan11, LLW12], where more examples are studied.

\section{Defect of the classical product}

\subsection{Cohomology correspondence for $P^{r}$ flops}

We recall the construction of ordinary flops in [LLW10] to fix the notation.

Let $X$ be a smooth complex projective manifold and $\psi: X \rightarrow \bar{X}$ a flopping contraction in the sense of minimal model theory, with $\bar{\psi}: Z \rightarrow S$ the restriction map on the exceptional loci. Assume that 
(i) $\bar{\psi}$ equips $Z$ with a $P^{r}$-bundle structure $\bar{\psi}: Z=P(F) \rightarrow S$ for some rank $r+1$ vector bundle $F$ over a smooth base $S$;

(ii) $\left.N_{Z / X}\right|_{Z_{s}} \cong \mathscr{O}_{P^{r}}(-1)^{\oplus(r+1)}$ for each $\bar{\psi}$-fiber $Z_{s}$ for $s \in S$.

Then there is another rank $r+1$ vector bundle $F^{\prime}$ over $S$ such that

$$
N_{Z / X} \cong \mathscr{O}_{P(F)}(-1) \otimes \bar{\psi}^{*} F^{\prime} .
$$

We may blow up $X$ along $Z$ to get $\phi: Y \rightarrow X$. The exceptional divisor

$$
E=P\left(N_{Z / X}\right) \cong P\left(\bar{\psi}^{*} F^{\prime}\right)=\bar{\psi}^{*} P\left(F^{\prime}\right)=P(F) \times{ }_{S} P\left(F^{\prime}\right)
$$

is a $P^{r} \times P^{r}$-bundle over $S$. We may then blow down $E$ along another fiber direction to get $\phi^{\prime}: Y \rightarrow X^{\prime}$. This induces another contraction $\psi^{\prime}: X^{\prime} \rightarrow \bar{X}$, with exceptional loci $\bar{\psi}^{\prime}: Z^{\prime}=$ $P\left(F^{\prime}\right) \rightarrow S$ and $\left.N_{Z^{\prime} / X^{\prime}}\right|_{\bar{\psi}^{\prime}-\text { fiber }} \cong \mathscr{O}_{P^{r}}(-1)^{\oplus(r+1)}$.

We call $f: X \rightarrow X^{\prime}$ an ordinary $P^{r}$ flop. The various sets and maps are summarized in the following commutative diagram:

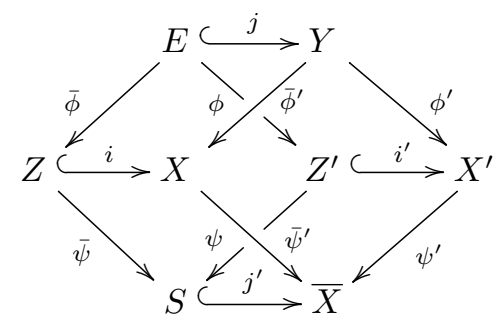

where the normal bundle of $E$ in $Y$ is

$$
N_{E / Y}=\bar{\phi}^{*} \mathscr{O}_{P(F)}(-1) \otimes \bar{\phi}^{* *} \mathscr{O}_{P\left(F^{\prime}\right)}(-1) \text {. }
$$

First of all, we have found a canonical correspondence between the cohomology groups of $X$ and $X^{\prime}$.

Theorem 2.1 ([LLW10]). For an ordinary $P^{r}$ flop $f: X \rightarrow X^{\prime}$, the graph closure $T:=\left[\bar{\Gamma}_{f}\right] \in$ $A\left(X \times X^{\prime}\right)$ identifies the Chow motives $\hat{X}$ of $X$ and $\hat{X}^{\prime}$ of $X^{\prime}$; that is, $\hat{X} \cong \hat{X}^{\prime}$ via $T^{t} \circ T=\Delta_{X}$ and $T \circ T^{t}=\Delta_{X^{\prime}}$. In particular, $\mathscr{F}:=T_{*}: H(X) \rightarrow H\left(X^{\prime}\right)$ preserves the Poincaré pairing on cohomology groups.

In practice, the correspondence $T$ induces a map on Chow groups:

$$
\mathscr{F}: A(X) \rightarrow A\left(X^{\prime}\right), \quad W \mapsto p_{*}^{\prime}\left(\bar{\Gamma}_{f} \cdot p^{*} W\right)=\phi_{*}^{\prime} \phi^{*} W,
$$

where $p$ and $p^{\prime}$ are the projection maps from $X \times X^{\prime}$ to $X$ and $X^{\prime}$, respectively.

Second, parallel to the procedure in [LLW10], we need to determine the explicit formulae for the associated map $\mathscr{F}$ restricted to $A(Z)$. The Leray-Hirsch theorem says that

$$
A(Z)=\bar{\psi}^{*} A(S)[h] / f_{F}(h),
$$

where $f_{F}(\lambda)=\lambda^{r+1}+\bar{\psi}^{*} c_{1}(F) \lambda^{r}+\cdots+\bar{\psi}^{*} c_{r+1}(F)$ is the Chern polynomial of $F$ and $h=$ $c_{1}\left(\mathscr{O}_{P(F)}(1)\right)$. Thus a class $\alpha \in A(Z)$ is of the form $\alpha=\sum_{i=0}^{r} h^{i} \bar{\psi}^{*} a_{i}$ for some $a_{i} \in A(S)$.

By the pull-back formula from intersection theory, it is easy to see that for $a \in A_{k}(Z)$ we have

$$
\phi^{*}\left(i_{*} a\right)=j_{*}\left(c_{r}(\mathscr{E}) \cdot \bar{\phi}^{*} a\right) \in A_{k}(Y)
$$




\section{INVARIANCE OF QUANTUM RINGS I}

where $\mathscr{E}$ is the excess normal bundle defined by

$$
0 \rightarrow N_{E / Y} \rightarrow \phi^{*} N_{Z / X} \rightarrow \mathscr{E} \rightarrow 0 .
$$

By the functoriality of the pull-back and push-forward, together with the above formula, we can conclude from $\mathscr{F}\left(i_{*}\left(\sum h^{i} \bar{\psi}^{*} a_{i}\right)\right)=\sum i_{*}^{\prime}\left(\mathscr{F}\left(i_{*}\left(h^{i}\right)\right) \cdot i_{*}^{\prime} \bar{\psi}^{\prime *} a_{i}\right)^{Z^{\prime}}$ that $\mathscr{F}$ restricted to $A(Z)$ is $A(S)$-linear. Here we identify the ring $A(S)$ with its isomorphic images in $A(Z)$ and $A\left(Z^{\prime}\right)$ via $\bar{\psi}^{*}$ and $\bar{\psi}^{\prime *}$, respectively.

Under such an identification, we will abuse the notation to denote $c_{i}(F), \bar{\psi}^{*} c_{i}(F)$ and $\bar{\psi}^{\prime *} c_{i}(F)$ by the same symbol $c_{i}$. Similarly, we denote $c_{i}\left(F^{\prime}\right), \bar{\psi}^{*} c_{i}\left(F^{\prime}\right)$ and $\bar{\psi}^{\prime *} c_{i}\left(F^{\prime}\right)$ by $c_{i}^{\prime}$. We use this abbreviation for any class in $A(S)$. And for $\alpha \in A(Z)$ we often omit $i_{*}$ from $i_{*} \alpha$ when $\alpha$ is regarded as a class in $A(X)$, unless possible confusion should arise. We do likewise for $\alpha^{\prime} \in A\left(Z^{\prime}\right) \hookrightarrow A\left(X^{\prime}\right)$.

The $A(S)$-linearity of $\mathscr{F}$ restricted to $A(Z)$ allows us to focus on the study of a basis for $A(Z)$ over $A(S)$. Recall that for a simple $P^{r}$ flop we have the basic transformation formula $\mathscr{F}\left(h^{k}\right)=(-1)^{r-k} h^{\prime k}$. Unfortunately, for a general $P^{r}$ flop, this does not hold anymore, so a better candidate has to be sought.

Note that the key ingredient in the pull-back formula is $c_{r}(\mathscr{E})$. From the Euler sequence

$$
0 \rightarrow \mathscr{O}_{Z^{\prime}}(-1) \rightarrow \bar{\psi}^{\prime *} F^{\prime} \rightarrow Q_{F^{\prime}} \rightarrow 0
$$

and the short exact sequence defining the excess normal bundle $\mathscr{E}$, we get $\mathscr{E}=\bar{\phi}^{*} \mathscr{O}_{P(F)}(-1) \otimes$ $\bar{\phi}^{\prime *} Q_{F^{\prime}}$. A simple computation leads to

$$
c_{r}(\mathscr{E})=(-1)^{r}\left(\bar{\phi}^{*} h^{r}-\bar{\phi}^{*} H_{1}^{\prime} \bar{\phi}^{*} h^{r-1}+\bar{\phi}^{\prime *} H_{2}^{\prime} \bar{\phi}^{*} h^{r-2}+\cdots+(-1)^{r} \bar{\phi}^{\prime *} H_{r}^{\prime}\right),
$$

where $H_{k}^{\prime}=c_{k}\left(Q_{F^{\prime}}\right)$. Explicitly,

$$
H_{k}^{\prime}=h^{\prime k}+c_{1}^{\prime} h^{\prime k-1}+\cdots+c_{k}^{\prime}
$$

where $h^{\prime}=c_{1}\left(\mathscr{O}_{P\left(F^{\prime}\right)}(1)\right)$. Similarly, we write

$$
H_{k}=c_{k}\left(Q_{F}\right)=h^{k}+c_{1} h^{k-1}+\cdots+c_{k} .
$$

Notice that $H_{k}=0=H_{k}^{\prime}$ for $k>r$. Finally, we find that $H_{k}$ and $H_{k}^{\prime}$ are the correct choices.

Proposition 2.2. For all positive integers $k \leqslant r$, we have $\mathscr{F}\left(H_{k}\right)=(-1)^{r-k} H_{k}^{\prime}$.

Proof. First of all, we have the basic identities $h^{r+1}+c_{1} h^{r}+\cdots+c_{r+1}=0, \bar{\phi}_{*}^{\prime} \bar{\phi}^{*} h^{i}=0$ for all $i<r$ and $\bar{\phi}_{*}^{\prime} \bar{\phi}^{*} h^{r}=\left[Z^{\prime}\right]$. The latter two follow from the definitions and dimension considerations.

In order to determine $\mathscr{F}\left(H_{k}\right)=\bar{\phi}_{*}^{\prime}\left(c_{r}(\mathcal{E}) \cdot \bar{\phi}^{*} H_{k}\right)$, we need to take care of the class

$$
\bar{\phi}_{*}^{\prime}\left(\bar{\phi}^{\prime *} H_{r-i}^{\prime} \bar{\phi}^{*} h^{i} \cdot \bar{\phi}^{*} H_{k}\right)
$$

with $0 \leqslant i \leqslant r$; here $H_{0}^{\prime}:=1$.

If $i>r-k$, then

$$
\begin{aligned}
\bar{\phi}_{*}^{\prime}\left(\bar{\phi}^{\prime *} H_{r-i}^{\prime} \bar{\phi}^{*} h^{i} \cdot \bar{\phi}^{*} H_{k}\right) & =\bar{\phi}_{*}^{\prime}\left(\bar{\phi}^{\prime *} H_{r-i}^{\prime} \bar{\phi}^{*}\left(h^{k+i}+c_{1} h^{k+i-1}+\cdots+c_{k} h^{i}\right)\right) \\
& =-\bar{\phi}_{*}^{\prime}\left(\bar{\phi}^{\prime *} H_{r-i}^{\prime} \bar{\phi}^{*}\left(c_{k+1} h^{i-1}+c_{k+2} h^{i-2}+\cdots+c_{r+1} h^{i+k-r-1}\right)\right)=0,
\end{aligned}
$$

since the power in $h$ is at most $i-1<r$.

If $i<r-k$, then again $\bar{\phi}_{*}^{\prime}\left(\bar{\phi}^{*} H_{r-i}^{\prime} \bar{\phi}^{*} h^{i} \cdot \bar{\phi}^{*} H_{k}\right)=0$, since the power in $h$ is at most $i+k<r$.

For the remaining case $i=r-k$,

$$
\bar{\phi}_{*}^{\prime}\left(\bar{\phi}^{\prime *} H_{r-i}^{\prime} \bar{\phi}^{*} h^{i} \cdot \bar{\phi}^{*} H_{k}\right)=\bar{\phi}_{*}^{\prime}\left(\bar{\phi}^{\prime *} H_{r-i}^{\prime} \bar{\phi}^{*} h^{r}\right)=H_{r-i}^{\prime}=H_{k}^{\prime} .
$$


We conclude that

$$
\mathscr{F}\left(H_{k}\right)=(-1)^{r} \sum_{i=0}^{r}(-1)^{r-i} \bar{\phi}_{*}^{\prime}\left(\bar{\phi}^{\prime *} H_{r-i}^{\prime} \bar{\phi}^{*} h^{i} \cdot \bar{\phi}^{*} H_{k}\right)=(-1)^{r-k} H_{k}^{\prime}
$$

Remark 2.3. The image class of $h^{k}$ under $\mathscr{F}$ looks more complicated here than in the case of simple $P^{r}$ flops. As a simple corollary of Proposition 2.2, we may show, by induction on $k$, that for all $k \in \mathbb{N}$,

$$
\mathscr{F}\left(h^{k}\right)=(-1)^{r-k}\left(a_{0} h^{\prime k}+a_{1} h^{\prime k-1}+\cdots+a_{k}\right) \in A\left(Z^{\prime}\right),
$$

where $a_{0}=1$ and the $a_{k} \in A(S)$ are determined by the recursive relations

$$
c_{k}^{\prime}=a_{k}-c_{1} a_{k-1}+c_{2} a_{k-2}+\cdots+(-1)^{k} c_{k} .
$$

Symmetrically,

$$
\mathscr{F}^{*}\left(h^{\prime k}\right)=(-1)^{r-k}\left(a_{0}^{\prime} h^{k}+a_{1}^{\prime} h^{k-1}+\cdots+a_{k}^{\prime}\right) \in A(Z)
$$

with $a_{0}^{\prime}=1$ and $a_{k}^{\prime}=c_{1}^{\prime} a_{k-1}^{\prime}-c_{2}^{\prime} a_{k-2}^{\prime}+\cdots+(-1)^{k-1} c_{k}^{\prime}+c_{k}$.

To put these formulae into perspective, we consider the virtual bundles

$$
A:=F^{\prime}-F^{*}, \quad A^{\prime}:=F-F^{*} .
$$

Then $a_{k}=c_{k}(A)$ and $a_{k}^{\prime}=c_{k}\left(A^{\prime}\right)$. Notice that since $a_{k}$ and $a_{k}^{\prime}$ are Chern classes of virtual bundles, they may survive even for $k \geqslant r+1$.

It is also interesting to notice that the explicit formula reduces to

$$
\mathscr{F}\left(h^{k}\right)=(-1)^{r-k} h^{\prime k},
$$

without lower-order terms precisely when $F^{\prime}$ equals $F^{*}$, the dual of $F$.

\subsection{Triple product}

Let $\left\{\bar{T}_{i}^{k}\right\}$ be a basis of $H^{2 k}(S)$ and $\left\{\check{\bar{T}}_{i}^{k}\right\} \subset H^{2(s-k)}(S)$ its dual basis, where $s=\operatorname{dim} S$. It is an easy but quite crucial discovery that the dual basis of the canonical basis $\left\{\bar{T}_{i}^{k} h^{j}\right\}$ in $H(Z)$ can be expressed in terms of $\left\{H_{k}\right\}_{k \geqslant 0}$.

LEMma 2.4. The dual basis of $\left\{\bar{T}_{i}^{k-j} h^{j}\right\}_{j \leqslant \min \{k, r\}}$ in $H^{2 k}(Z)$ is $\left\{\check{\bar{T}}_{i}^{k-j} H_{r-j}\right\}_{j \leqslant \min \{k, r\}}$ in $H^{2(r+s-k)}(Z)$.

Proof. We have to check that $\left(\bar{T}_{i}^{k-j} h^{j} \cdot \check{\bar{T}}_{i}^{k-j} H_{r-j}\right)=1$ and $\left(\bar{T}_{i}^{k-j} h^{j} \cdot \check{\bar{T}}_{i}^{k-j^{\prime}} H_{r-j^{\prime}}\right)=0$ for any $j \neq j^{\prime}$. Indeed,

$$
\left(\bar{T}_{i}^{k-j} h^{j} \cdot \check{\bar{T}}_{i}^{k-j} H_{r-j}\right)=\bar{T}^{s}\left(h^{r}+c_{1} h^{r-1}+\cdots\right)=\bar{T}^{s} h^{r}=1,
$$

since $\bar{T}^{s} c_{i}=0$ for all $i \geqslant 1$ by degree considerations.

Notation 2.5. When $X$ is a bundle over $S$, classes in $H(S)$ may be considered as classes in $H(X)$ by the obvious pull-back, which we often omit in the notation. To avoid confusion, we consistently employ the notation $\check{\bar{T}}_{i}$ as the dual class of $\bar{T}_{i} \in H(S)$ with respect to the Poincaré pairing in $S$. The "raised" index form, for example $T^{\mu}$ as the dual of $T_{\mu} \in H(X)$, is reserved for duality with respect to the Poincaré pairing in $X$.

Now if $j^{\prime}>j$, then

$$
k-j+\left(s-\left(k-j^{\prime}\right)\right)=s+\left(j^{\prime}-j\right)>s,
$$




\section{INVARIANCE OF QUANTUM RINGS I}

which implies $\bar{T}_{i}^{k-j} \check{\bar{T}}_{i}^{k-j^{\prime}}=0$. Conversely, if $j^{\prime}<j$, then $\bar{T}_{i}^{k-j} \check{\bar{T}}_{i}^{k-j^{\prime}} \in H^{2\left(s-\left(j-j^{\prime}\right)\right)}(S)$ and

$$
\begin{aligned}
h^{j} H_{r-j^{\prime}} & =h^{r+\left(j-j^{\prime}\right)}+c_{1} h^{r+\left(j-j^{\prime}\right)-1}+\cdots+c_{r-j^{\prime}} h^{j} \\
& =-c_{r-j^{\prime}+1} h^{j-1}-\cdots-c_{r+1} h^{j-j^{\prime}-1} .
\end{aligned}
$$

Again, since

$$
\left(s-\left(j-j^{\prime}\right)\right)+\left(r-j^{\prime}+z\right)=s+(r+z-j)>s
$$

for $z \geqslant 1$, we have $\bar{T}_{i}^{k-j} \check{\bar{T}}_{i}^{k-j^{\prime}} c_{r-j^{\prime}+z} h^{j-z-1}=0$. The result follows.

Now we can determine the difference of the pull-backs of the classes $a$ and $\mathscr{F} a$ as follows.

Proposition 2.6. For a class $a \in H^{2 k}(X)$, let $a^{\prime}=\mathscr{F} a$ in $X^{\prime}$. Then

$$
\phi^{*} a^{\prime}=\phi^{*} a+j_{*} \sum_{i} \sum_{1 \leqslant j \leqslant \min \{k, r\}}\left(a \cdot \check{\bar{T}}_{i}^{k-j} H_{r-j}\right) \bar{T}_{i}^{k-j} \frac{x^{j}-(-y)^{j}}{x+y},
$$

where $x=\bar{\phi}^{*} h$ and $y=\bar{\phi}^{* *} h^{\prime}$.

Proof. Recall that

$$
N_{E / Y}=\bar{\phi}^{*} \mathscr{O}_{Z}(-1) \otimes \bar{\phi}^{\prime *} \mathscr{O}_{Z^{\prime}}(-1),
$$

and hence $c_{1}\left(N_{E / Y}\right)=-(x+y)$. Since the difference $\phi^{\prime *} a^{\prime}-\phi^{*} a$ has support in $E$, we may write $\phi^{\prime *} a^{\prime}-\phi^{*} a=j_{*} \lambda$ for some $\lambda \in H^{2(k-1)}(E)$. Then

$$
\left.\left(\phi^{*} a^{\prime}-\phi^{*} a\right)\right|_{E}=j^{*} j_{*} \lambda=c_{1}\left(N_{E / Y}\right) \lambda=-(x+y) \lambda .
$$

Notice that while the inclusion-restriction map $j^{*} j_{*}$ on $H(E)$ may have non-trivial kernel, elements in the kernel never occur in $\phi^{*} a^{\prime}-\phi^{*} a$, by the Chow moving lemma. Indeed, if $j^{*} j_{*} \lambda \equiv$ $\left.j_{*} \lambda\right|_{E}=0$, then $j_{*} \lambda$ is rationally equivalent to a cycle $\lambda^{\prime}$ disjoint from $E$. Applying $\phi_{*}^{\prime}$ to the equation

$$
\phi^{*} a^{\prime}-\phi^{*} a=j_{*} \lambda \sim \lambda^{\prime}
$$

gives rise to

$$
\phi_{*}^{\prime} \lambda^{\prime} \sim \phi_{*}^{\prime} \phi^{*} a^{\prime}-\phi_{*}^{\prime} \phi^{*} a=a^{\prime}-a^{\prime}=0 .
$$

This leads to $\lambda^{\prime} \sim 0$ on $Y$.

Hence

$$
\lambda=-\frac{1}{x+y}\left(\left.\left(\phi^{\prime *} a^{\prime}\right)\right|_{E}-\left.\left(\phi^{*} a\right)\right|_{E}\right)=-\frac{1}{x+y}\left(\bar{\phi}^{\prime *}\left(\left.a^{\prime}\right|_{Z^{\prime}}\right)-\bar{\phi}^{*}\left(\left.a\right|_{Z}\right)\right) .
$$

By Lemma 2.4, we get

$$
\begin{aligned}
\bar{\phi}^{*}\left(\left.a\right|_{Z}\right) & =\bar{\phi}^{*}\left(\sum_{i} \sum_{j \leqslant \min \{k, r\}}\left(a \cdot \check{\bar{T}}_{i}^{k-j} H_{r-j}\right) \bar{T}_{i}^{k-j} h^{j}\right) \\
& =\sum_{i} \sum_{j \leqslant \min \{k, r\}}\left(a \cdot \check{\bar{T}}_{i}^{k-j} H_{r-j}\right) \bar{T}_{i}^{k-j} x^{j} .
\end{aligned}
$$

Similarly, we have

$$
\bar{\phi}^{\prime *}\left(\left.a^{\prime}\right|_{Z^{\prime}}\right)=\sum_{i} \sum_{j \leqslant \min \{k, r\}}\left(a^{\prime} \cdot \check{\bar{T}}_{i}^{k-j} H_{r-j}^{\prime}\right) \bar{T}_{i}^{k-j} y^{j} .
$$

Since $\mathscr{F}$ preserves the Poincaré pairing, we have

$$
\left(a^{\prime} \cdot \check{\bar{T}}_{i}^{k-j} H_{r-j}^{\prime}\right)=\left(\mathscr{F} a \cdot \mathscr{F}\left((-1)^{r-(r-j)} \check{\bar{T}}_{i}^{k-j} H_{r-j}\right)\right)=(-1)^{j}\left(a \cdot \check{\bar{T}}_{i}^{k-j} H_{r-j}\right) .
$$


Putting these together, we obtain

$$
\lambda=\sum_{i} \sum_{1 \leqslant j \leqslant \min \{k, r\}}\left(a \cdot \check{\bar{T}}_{i}^{k-j} H_{r-j}\right) \bar{T}_{i}^{k-j} \frac{x^{j}-(-y)^{j}}{x+y} .
$$

Remark 2.7. Notice that since the power in $x$ (and in $y$ ) is at most $r-1$, the class $\lambda$ clearly contains non-trivial fiber directions of both morphisms $\bar{\phi}$ and $\bar{\phi}^{\prime}$. Thus this proposition in particular gives rise to an alternative proof of the equivalence of Chow motives under ordinary flops (Theorem 3.6). Indeed, this is precisely the quantitative version of the original proof in [LLW10].

Now, we may compare the triple products of classes in $X$ and $X^{\prime}$.

Theorem 2.8. Let $a_{i} \in H^{2 k_{i}}(X)$ for $i=1,2,3$ with $k_{1}+k_{2}+k_{3}=\operatorname{dim} X=s+2 r+1$. Then

$$
\begin{aligned}
\left(\mathscr{F} a_{1} \cdot \mathscr{F} a_{2} \cdot \mathscr{F} a_{3}\right)=\left(a_{1} \cdot a_{2} \cdot a_{3}\right)+ & (-1)^{r} \sum\left(a_{1} \cdot \check{\bar{T}}_{i_{1}}^{k_{1}-j_{1}} H_{r-j_{1}}\right)\left(a_{2} \cdot \check{\bar{T}}_{i_{2}}^{k_{2}-j_{2}} H_{r-j_{2}}\right) \\
& \times\left(a_{3} \cdot \check{\bar{T}}_{i_{3}}^{k_{3}-j_{3}} H_{r-j_{3}}\right)\left(\tilde{s}_{j_{1}+j_{2}+j_{3}-2 r-1} \bar{T}_{i_{1}}^{k_{1}-j_{1}} \bar{T}_{i_{2}}^{k_{2}-j_{2}} \bar{T}_{i_{3}}^{k_{3}-j_{3}}\right),
\end{aligned}
$$

where the sum is over all possible $i_{1}, i_{2}, i_{3}$ and $j_{1}, j_{2}, j_{3}$ subject to the constraints $1 \leqslant j_{p} \leqslant$ $\min \left\{r, k_{p}\right\}$ for $p=1,2,3$ and $j_{1}+j_{2}+j_{3} \geqslant 2 r+1$. Here

$$
\tilde{s}_{i}:=s_{i}\left(F+F^{*}\right)
$$

is the ith Segre class of $F+F^{* *}$.

Proof. First of all, $\phi^{*} \mathscr{F} a_{i}=\phi^{*} a_{i}+j_{*} \lambda_{i}$ for some $\lambda_{i} \in H^{2\left(k_{i}-1\right)}(E)$ which contains both fiber directions $\bar{\phi}$ and $\bar{\phi}^{\prime}$. Hence

$$
\begin{aligned}
& \left(\mathscr{F} a_{1} \cdot \mathscr{F} a_{2} \cdot \mathscr{F} a_{3}\right)=\left(\phi^{*} \mathscr{F} a_{1} \cdot \phi^{*} \mathscr{F} a_{2} \cdot\left(\phi^{*} a_{3}+j_{*} \lambda_{3}\right)\right)=\left(\phi^{*} \mathscr{F} a_{1} \cdot \phi^{*} \mathscr{F} a_{2} \cdot \phi^{*} a_{3}\right) \\
& =\left(\left(\phi^{*} a_{1}+j_{*} \lambda_{1}\right) \cdot\left(\phi^{*} a_{2}+j_{*} \lambda_{2}\right) \cdot \phi^{*} a_{3}\right) .
\end{aligned}
$$

When we expand this, the first term is clearly equal to $\left(a_{1} \cdot a_{2} \cdot a_{3}\right)$.

For those terms with two pull-backs like $\phi^{*} a_{1} \cdot \phi^{*} a_{3}$, the intersection values are zero since the remaining part necessarily contains a non-trivial fiber direction of $\bar{\phi}$.

The terms with $\phi^{*} a_{3}$ and two exceptional parts contribute sums over $i_{1}, i_{2}, j_{1}$ and $j_{2}$ of products of

$$
\begin{aligned}
\phi^{*} a_{3} & \cdot j_{*} \bar{T}_{i_{1}}^{k_{1}-j_{1}}\left(\frac{x^{j_{1}}-(-y)^{j_{1}}}{x+y}\right) \cdot j_{*} \bar{T}_{i_{2}}^{k_{2}-j_{2}}\left(\frac{x^{j_{2}}-(-y)^{j_{2}}}{x+y}\right) \\
& =-\phi^{*} a_{3} \cdot j_{*}\left(\bar{T}_{i_{1}}^{k_{1}-j_{1}} \bar{T}_{i_{2}}^{k_{2}-j_{2}}\left(x^{j_{1}}-(-y)^{j_{1}}\right)\left(x^{j_{2}-1}+x^{j_{2}-2}(-y)+\cdots+(-y)^{j_{2}-1}\right)\right)
\end{aligned}
$$

and $\left(a_{1} \cdot \check{\bar{T}}_{i_{1}}^{k_{1}-j_{1}} H_{r-j_{1}}\right)\left(a_{2} \cdot \check{\bar{T}}_{i_{2}}^{k_{2}-j_{2}} H_{r-j_{2}}\right)$. The terms with non-trivial contribution must contain $y^{q}$ with $q \geqslant r$, which implies $j_{1}+j_{2}-1 \geqslant r$; hence these terms are

$$
-(-y)^{j_{1}}\left(x^{j_{2}-1-\left(r-j_{1}\right)}(-y)^{r-j_{1}}+x^{j_{2}-1-\left(r-j_{1}\right)-1}(-y)^{r-j_{1}+1}+\cdots+(-y)^{j_{2}-1}\right),
$$

and their contribution after taking $\phi_{*}$ is

$$
(-1)^{r+1}\left(h^{j_{1}+j_{2}-r-1}-h^{j_{1}+j_{2}-r-2} s_{1}^{\prime}+\cdots+(-1)^{j_{1}+j_{2}-r-1} s_{j_{1}+j_{2}-r-1}^{\prime}\right),
$$

where $s_{i}^{\prime}:=s_{i}\left(F^{\prime}\right)$ is the $i$ th Segre class of $F^{\prime}$. Here we use the property of Segre classes to obtain $\phi_{*} y^{q}=s_{q-r}^{\prime}$ for $q \geqslant r+1$. 


\section{INVARIANCE OF QUANTUM RINGS I}

In a bundle-theoretic formulation,

$$
\begin{aligned}
& h^{j_{1}+j_{2}-r-1}-h^{j_{1}+j_{2}-r-2} s_{1}^{\prime}+\cdots+(-1)^{j_{1}+j_{2}-r-1} s_{j_{1}+j_{2}-r-1}^{\prime} \\
& \quad=\left(\left(1-s_{1}^{\prime}+s_{2}^{\prime}+\cdots\right)\left(1+h+h^{2}+\cdots\right)\right)_{j_{1}+j_{2}-r-1} \\
& \quad=\left(s\left(F^{\prime *}\right) \frac{1}{(1-h)}\right)_{j_{1}+j_{2}-r-1}=\left(\frac{c(F)}{(1-h)} s(F) s\left(F^{\prime *}\right)\right)_{j_{1}+j_{2}-r-1} \\
& \quad=\left(c\left(Q_{F}\right) \cdot s\left(F+F^{\prime *}\right)\right)_{j_{1}+j_{2}-r-1} \\
& \quad=H_{j_{1}+j_{2}-r-1}+H_{j_{1}+j_{2}-r-2} \tilde{s}_{1}+\cdots+\tilde{s}_{j_{1}+j_{2}-r-1} .
\end{aligned}
$$

With respect to the basis $\left\{\check{\bar{T}}_{i}^{k}\right\}$, the term $\tilde{s}_{p} \bar{T}_{i_{1}}^{k_{1}-j_{1}} \bar{T}_{i_{2}}^{k_{2}-j_{2}}$ is of the form

$$
\sum_{i_{3}}\left(\tilde{s}_{p} \bar{T}_{i_{1}}^{k_{1}-j_{1}} \bar{T}_{i_{2}}^{k_{2}-j_{2}} \bar{T}_{i_{3}}^{k_{3}-\left(2 r+1+p-j_{1}-j_{2}\right)}\right) \check{\bar{T}}_{i_{3}}^{k_{3}-\left(2 r+1+p-j_{1}-j_{2}\right)} .
$$

We define the new index $j_{3}=2 r+1+p-j_{1}-j_{2}$; thus $j_{1}+j_{2}+j_{3} \geqslant 2 r+1$ and $p=$ $j_{1}+j_{2}+j_{3}-2 r-1$.

By summing all together, we get the result.

There is a particularly simple case where no $H_{i}$ or Segre classes $\tilde{s}_{i}$ are needed in the defect formula, namely the $P^{1}$ flops.

Corollary 2.9. For $P^{1}$ flops over any smooth base $S$ of dimension $s$, consider $a_{i} \in H^{2 k_{i}}(X)$ for $i=1,2,3$ with $k_{1}+k_{2}+k_{3}=\operatorname{dim} X=s+3$. Then

$$
\left(\mathscr{F} a_{1} \cdot \mathscr{F} a_{2} \cdot \mathscr{F} a_{3}\right)=\left(a_{1} \cdot a_{2} \cdot a_{3}\right)-\sum\left(a_{1} \cdot \check{\bar{T}}_{1}\right)\left(a_{2} \cdot \check{T}_{2}\right)\left(a_{3} \cdot \check{\bar{T}}_{3}\right)\left(\bar{T}_{1} \bar{T}_{2} \bar{T}_{3}\right)
$$

with the $\bar{T}_{i}$ running over all classes in the chosen basis of $H^{2\left(k_{i}-1\right)}(S)$.

There is a trivial but useful observation on when the product is preserved.

Corollary 2.10. For a $P^{r}$ flop $f: X \rightarrow X^{\prime}$ and classes $a_{1} \in H^{2 k_{1}}(X)$ and $a_{2} \in H^{2 k_{2}}(X)$ with $k_{1}+k_{2} \leqslant r$, we have $\mathscr{F}\left(a_{1} \cdot a_{2}\right)=\mathscr{F} a_{1} \cdot \mathscr{F} a_{2}$.

This follows from Theorem 2.8, since all correction terms vanish for any $a_{3}$. In fact, it is a consequence of a dimension count.

\section{Quantum corrections attached to the extremal ray}

\subsection{The set-up with non-trivial base}

Let $a_{i} \in H^{2 k_{i}}(X)$ for $i=1, \ldots, n$ with

$$
\sum_{i=1}^{n} k_{i}=2 r+1+s+(n-3) .
$$

Since

$$
\left.a_{i}\right|_{Z}=\sum_{s_{i}} \sum_{j_{i} \leqslant \min \left\{k_{i}, r\right\}}\left(a_{i} \cdot \check{\bar{T}}_{s_{i}}^{k_{i}-j_{i}} H_{r-j_{i}}\right) \bar{T}_{s_{i}}^{k_{i}-j_{i}} h^{j_{i}}
$$


we compute

$$
\begin{aligned}
\left\langle a_{1}, \ldots, a_{n}\right\rangle_{0, n, d \ell}^{X} & =\sum_{\vec{s}, \vec{j}} \int_{\bar{M}_{0, n}(Z, d \ell)} \prod_{i=1}^{n}\left(\left(a_{i} \cdot \check{T}_{s_{i}}^{k_{i}-j_{i}} H_{r-j_{i}}\right) e_{i}^{*}\left(\bar{\psi}^{*} \bar{T}_{s_{i}}^{k_{i}-j_{i}} \cdot h^{j_{i}}\right)\right) \cdot e\left(R^{1} f t_{*} e_{n+1}^{*} N\right) \\
& =\sum_{\vec{s}, \vec{j}} \prod_{i=1}^{n}\left(a_{i} \cdot \check{\bar{T}}_{s_{i}}^{k_{i}-j_{i}} H_{r-j_{i}}\right)\left[\prod_{i=1}^{n} \bar{T}_{s_{i}}^{k_{i}-j_{i}} \cdot \Psi_{n *}\left(\prod_{i=1}^{n} e_{i}^{*} h^{j_{i}} \cdot e\left(R^{1} f t_{*} e_{n+1}^{*} N\right)\right)\right]^{S},
\end{aligned}
$$

where the sum is taken over all $\vec{s}=\left(s_{1}, \ldots, s_{n}\right)$ and all admissible $\vec{j}=\left(j_{1}, \ldots, j_{n}\right)$. By the fundamental class axiom, we must have $j_{i} \geqslant 1$ for all $i$.

Here we make use of

$$
\left[\bar{M}_{0, n}(X, d \ell)\right]^{\text {virt }}=\left[\bar{M}_{0, n}(Z, d \ell)\right] \cap e\left(R^{1} f t_{*} e_{n+1}^{*} N\right)
$$

and the fiber bundle diagram over $S$

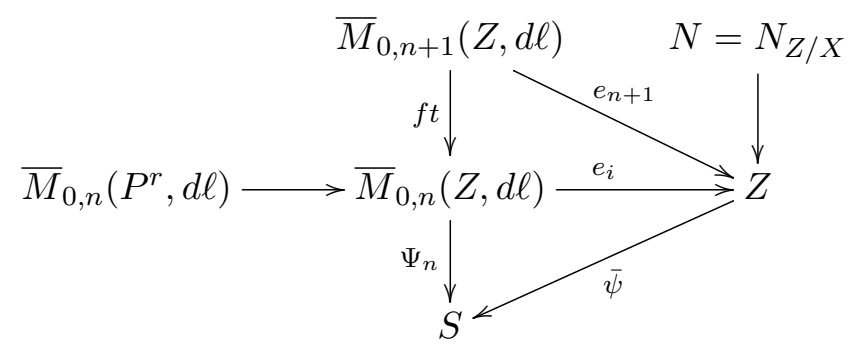

as well as the fact that classes in $S$ are constants among the bundle morphisms (by the projection formula applied to $\Psi_{n}=\bar{\psi} \circ e_{i}$ for each $\left.i\right)$.

We must have $\sum\left(k_{i}-j_{i}\right) \leqslant s$ to get non-trivial invariants. That is,

$$
\sum_{i=1}^{n} j_{i} \geqslant 2 r+1+n-3 .
$$

If the equality holds, then $\prod_{i=1}^{n} \bar{T}_{s_{i}}^{k_{i}-j_{i}}$ is a zero-dimensional cycle in $S$ and the invariant readily reduces to the corresponding one on any fiber, namely the simple case, which is completely determined in [LLW10]:

$$
\left(\bar{T}_{s_{1}}^{k_{1}-j_{1}} \ldots \bar{T}_{s_{n}}^{k_{n}-j_{n}}\right)^{S}\left\langle h^{j_{1}}, \ldots, h^{j_{n}}\right\rangle_{0, n, d \ell}^{\text {simple }}=\left(\prod \bar{T}_{s_{i}}^{k_{1}-j_{1}}\right)^{S} N_{\vec{j}} d^{n-3}(-1)^{(d-1)(n+1)} .
$$

If, on the contrary, the strict inequality holds, then by dimension counting in the simple case, the restriction of the fiber integral $\Psi_{n *}(\cdot)$ to points in $S$ vanishes. In fact, the fiber integral is represented by a cycle $S_{\vec{j}} \subset S$ with codimension

$$
\nu:=\sum j_{i}-(2 r+1+n-3) .
$$

The structure of $S_{\vec{j}}$ necessarily depends on the bundles $F$ and $F^{\prime}$.

One would expect the end formula for $\Psi_{n *}(\cdot)$ to be

$$
s_{\nu}\left(F+F^{\prime *}\right) N_{\vec{j}} d^{n-3}
$$

with $N_{\vec{j}}=1$ for $n \leqslant 3$, so that the difference of the corresponding generating functions on $X$ and $X^{\prime}$ cancels out with the classical defect on the cup product. Unfortunately, the actual behavior of these Gromov-Witten invariants with base dimension $s>0$ is more delicate than this. 


\section{INVARIANCE OF QUANTUM RINGS I}

Notice that the new phenomenon does not occur for $n=2$. In that case, $k_{1}+k_{2}=2 r+s$ and $j_{1}=j_{2}=r$, and we may assume that $\bar{T}_{s_{2}}$ runs through the dual basis of $\bar{T}_{s_{1}}$. Since then the non-trivial terms only appear when $\bar{T}_{s_{1}}$ and $\bar{T}_{s_{2}}$ are dual to each other, we get

$$
\begin{aligned}
\left\langle a_{1}, a_{2}\right\rangle_{0,2, d \ell}^{X} & =\sum_{s}\left(a_{1} \cdot \bar{T}_{s}\right)\left(a_{2} \cdot \check{\bar{T}}_{s}\right)\left\langle h^{r}, h^{r}\right\rangle_{d}^{\text {simple }} \\
& =(-1)^{(d-1)(r+1)} \frac{1}{d} \sum_{s}\left(a_{1} \cdot \bar{T}_{s}\right)\left(a_{2} \cdot \check{\bar{T}}_{s}\right) .
\end{aligned}
$$

It is also clear that the new phenomenon does not occur for $P^{1}$ flops over an arbitrary smooth base $S$. Thus, before dealing with the general cases, we will work out the first (simplest) new case to demonstrate the general picture that will occur.

\subsection{Twisted relative invariants for $\nu=1$}

Consider $P^{r}$ flops with $n=3$ and $j_{1}+j_{2}+j_{3}=(2 r+1)+1=2 r+2$, namely with degree 1 more (that is, $\nu=1)$ than the old case. We start with $\left(j_{1}, j_{2}, j_{3}\right)=(2, r, r)$. Since classes from $S$ can be merged into any marked point, the invariant to be taken care of is

$$
\left\langle h^{2}, h^{r}, \bar{t} h^{r}\right\rangle_{d}^{X}
$$

for some $\bar{t} \in H^{2(s-1)}(S)$. Equivalently, we define the fiber integral

$$
\left\langle\prod_{i=1}^{n} h^{j_{i}}\right\rangle_{d}^{/ S}:=\Psi_{n *}\left(\prod_{i=1}^{n} e_{i}^{*} h^{j_{i}} \cdot e\left(R^{1} f t_{*} e_{n+1}^{*} N\right)\right) \in A(S)
$$

to be a $\bar{\psi}$-relative invariant over $S$, and we are now computing

$$
\left\langle h^{2}, h^{r}, \bar{t} h^{r}\right\rangle_{d}^{X}=\left(\left\langle h^{2}, h^{r}, h^{r}\right\rangle_{d}^{/ S} \cdot \bar{t}\right)^{S} .
$$

Notice that for $r=2$, we have $6 \geqslant j_{1}+j_{2}+j_{3}>5$; hence $(2,2,2)$ is the only new case to compute.

The basic idea is to use the divisor relation [LP04] (for $n$-point invariants with $n \geqslant 3$ )

$$
e_{i}^{*} h=e_{j}^{*} h+\sum_{d^{\prime}+d^{\prime \prime}=d}\left(d^{\prime \prime}\left[D_{i k, d^{\prime} \mid j, d^{\prime \prime}}\right]^{\text {virt }}-d^{\prime}\left[D_{i, d^{\prime} \mid j k, d^{\prime \prime}}\right]^{\text {virt }}\right)
$$

to move various classes $h$ to the same marked point. This type of process is also referred to as divisorial reconstruction in this paper. Once the power exceeds $r$, the Chern polynomial relation reduces $h^{r+1}$ to lower-degree powers coupled with (Chern) classes from the base $S$. This will eventually reduce the new invariants to old cases. While this procedure is well known as the reconstruction principle in Gromov-Witten theory, the moral here is to show that this reconstruction transforms perfectly under flops.

Let $\Delta(X)=\sum_{\mu} T_{\mu} \otimes T^{\mu}$ be a diagonal splitting of $\Delta(X) \subset X \times X$. That is, $\left\{T_{\mu}\right\}$ is a cohomology basis of $H(X)$ with dual basis $\left\{T^{\mu}\right\}$. Applying the divisor relation (3.1), we get

$$
\left\langle h^{2}, h^{r}, \bar{t} h^{r}\right\rangle_{d}=\left\langle h, h^{r+1}, \bar{t} h^{r}\right\rangle_{d}+\sum_{d^{\prime}+d^{\prime \prime}=d} \sum_{\mu} d^{\prime \prime}\left\langle h, \bar{t} h^{r}, T_{\mu}\right\rangle_{d^{\prime}}\left\langle T^{\mu}, h^{r}\right\rangle_{d^{\prime \prime}}-d^{\prime}\left\langle h, T_{\mu}\right\rangle_{d^{\prime}}\left\langle T^{\mu}, h^{r}, \bar{t} h^{r}\right\rangle_{d^{\prime \prime}} .
$$

The last term in the sum vanishes since there are no (non-trivial) 2-point invariants of the form $\left\langle h, T_{\mu}\right\rangle_{d^{\prime}}$.

Since $h^{r+1}=-c_{1} h^{r}-c_{2} h^{r-1}-\cdots-c_{r+1}$, the first term clearly equals

$$
-\left(c_{1} \cdot \bar{t}\right)^{S}\left\langle h, h^{r}, h^{r}\right\rangle_{d}^{\text {simple }}=-(-1)^{(d-1)(r+1)}\left(c_{1} \cdot \bar{t}\right)^{S} .
$$


For the second term, notice that the only degree zero invariant is given by the 3 -point classical cup product. Hence if $d^{\prime}=0$, then we may select $\left\{T^{\mu}\right\}$ in such a way that $h \cdot \bar{t} h^{r}$ appears as one of the basis elements, say $T^{0}=\bar{t} h^{r+1}$ (this is not part of the canonical basis). Thus $d^{\prime \prime}=d$ and the term equals

$$
d\left\langle h, \bar{t} h^{r}, T_{0}\right\rangle_{0}\left\langle\bar{t} h^{r+1}, h^{r}\right\rangle_{d}=-d\left(c_{1} \cdot \bar{t}\right)^{S}\left\langle h^{r}, h^{r}\right\rangle_{d}^{\text {simple }}=-(-1)^{(d-1)(r+1)}\left(c_{1} \cdot \bar{t}\right)^{S} .
$$

It remains to consider the case $1 \leqslant d^{\prime \prime} \leqslant d-1$. In this case we may assume $T_{0}=\check{t} h^{r}$, since no lower power in $h$ is allowed. Since we are considering extremal rays, to compute $T^{0}$ explicitly we may work on the projective local model $X_{\text {loc }}=P\left(N_{Z / X} \oplus \mathscr{O}\right)$ of $X$ along $Z$.

By applying Lemma 2.4 to $H\left(X_{\text {loc }}\right)$, we get the following result.

LEMma 3.1. Let $\left\{z_{i}\right\}$ be a basis of $H(Z)$ and $\xi=c_{1}\left(\mathscr{O}_{P(N \oplus \mathscr{O})}(1)\right)$ the class of the infinity divisor $E$. The dual basis for $\left\{z_{i} \xi^{r+1-j}\right\}_{j \leqslant r+1}$ is given by $\left\{\check{z}_{i} \Theta_{j}\right\}_{j \leqslant r+1}$, where

$$
\Theta_{j}:=c_{j}\left(Q_{N}\right)=\xi^{j}+c_{1}(N) \xi^{j-1}+\cdots+c_{j}(N) .
$$

In particular, $\left.\Theta_{j}\right|_{Z}=c_{j}(N)$. Moreover, since $N=\bar{\psi}^{*} F^{\prime} \otimes \mathscr{O}(-1)$, we have

$$
c_{r+1}(N)=(-1)^{r+1}\left(h^{r+1}-c_{1}^{\prime} h^{r}+\cdots+(-1)^{r+1} c_{r+1}^{\prime}\right) .
$$

Now, if $z_{0}=\check{\bar{t}} h^{r}$ and $T_{0}=z_{0} \xi^{0}=\check{\bar{t}} h^{r}$, then $T^{0}=\bar{t} \Theta_{r+1}$ and the invariants become

$$
\begin{aligned}
d^{\prime \prime}\left\langle h, \bar{t} h^{r}, \check{\bar{t}} h^{r}\right\rangle_{d^{\prime}}\left\langle\bar{t} c_{r+1}(N), h^{r}\right\rangle_{d^{\prime \prime}} & =-(-1)^{\left(d^{\prime}-1\right)(r+1)}(-1)^{r+1} d^{\prime \prime}\left(\bar{t} \cdot\left(c_{1}+c_{1}^{\prime}\right)\right)^{S}\left\langle h^{r}, h^{r}\right\rangle_{d^{\prime \prime}}^{\text {simple }} \\
& =-(-1)^{\left(d^{\prime}-1+d^{\prime \prime}-1+1\right)(r+1)}\left(\left(c_{1}+c_{1}^{\prime}\right) \cdot \bar{t}\right)^{S} \\
& =-(-1)^{(d-1)(r+1)}\left(\left(c_{1}+c_{1}^{\prime}\right) \cdot \bar{t}\right)^{S} .
\end{aligned}
$$

Summing these together, we get

$$
\left\langle h^{2}, h^{r}, \bar{t} h^{r}\right\rangle_{d}=(-1)^{(d-1)(r+1)}\left(\left(\left(-c_{1}+c_{1}^{\prime}\right) \cdot \bar{t}\right)^{S}-d\left(\left(c_{1}+c_{1}^{\prime}\right) \cdot \bar{t}\right)^{S}\right) .
$$

By exactly the same procedure, as long as $j_{2}<r$ or $j_{3}<r$, the boundary terms in the divisor relation necessarily vanish, as we can deduce from our knowledge of 2-point invariants, hence

$$
\left\langle h^{j_{1}}, h^{j_{2}}, \bar{t} h^{j_{3}}\right\rangle_{d}=\left\langle h^{j_{1}-1}, h^{j_{2}+1}, \bar{t} h^{j_{3}}\right\rangle_{d} .
$$

In particular, any invariant with $j_{1}+j_{2}+j_{3}=2 r+2$ may be inductively transformed into $\left\langle h^{2}, h^{r}, \bar{t} h^{r}\right\rangle_{d}$. Hence we have shown the following result.

Proposition $3.2(n=3, \nu=1)$. For $\sum_{i=1}^{3} j_{i}=2 r+2$ and $\bar{t} \in H^{2(s-1)}(S)$,

$$
\left\langle h^{j_{1}}, h^{j_{2}}, \bar{t} h^{j_{3}}\right\rangle_{d}=(-1)^{(d-1)(r+1)}\left(\left(\tilde{s}_{1} \cdot \bar{t}\right)^{S}-d\left(c_{1}\left(F+F^{\prime}\right) \cdot \bar{t}\right)^{S}\right) .
$$

As in [LLW10], this implies that the 3-point extremal quantum corrections for $X$ and $X^{\prime}$ remedy the defect of the classical cup product for the case $\nu=1$.

To see this, it is convenient to consider the basic rational function

$$
\mathbf{f}(q):=\frac{q}{1-(-1)^{r+1} q}=\sum_{d \geqslant 1}(-1)^{(d-1)(r+1)} q^{d},
$$

which is the 3-point extremal correction for the case $\nu=0$. It is clear that

$$
\mathbf{f}(q)+\mathbf{f}\left(q^{-1}\right)=(-1)^{r} .
$$

Since $\mathscr{F}\left(\bar{t} h^{j}\right)=(-1)^{j} \bar{t} h^{\prime j}$ for $j \leqslant r$, the geometric series on $X$

$$
\sum_{d \geqslant 1}(-1)^{(d-1)(r+1)}\left(\tilde{s}_{1} \cdot \bar{t}\right)^{S} q^{d \ell}=\left(\tilde{s}_{1} \cdot \bar{t}\right)^{S} \mathbf{f}\left(q^{\ell}\right),
$$




\section{INVARIANCE OF QUANTUM RINGS I}

together with its counterpart on $X^{\prime}$, exactly corrects the classical term via

$$
\left(\tilde{s}_{1} \cdot \bar{t}\right)^{S} \mathbf{f}\left(q^{\ell}\right)-(-1)^{j_{1}+j_{2}+j_{3}}\left(\tilde{s}_{1}^{\prime} \cdot \bar{t}\right)^{S} \mathbf{f}\left(q^{\ell^{\prime}}\right)=\left(\tilde{s}_{1} \cdot \bar{t}\right)^{S}\left(\mathbf{f}\left(q^{\ell}\right)+\mathbf{f}\left(q^{-\ell}\right)\right)=(-1)^{r}\left(\tilde{s}_{1} \cdot \bar{t}\right)^{S} .
$$

The new feature for $\nu=1$ is that we also have contributions involving the differential operator $\delta_{h}=q^{\ell} d / d q^{\ell}$, namely

$$
-\left(c_{1}\left(F+F^{\prime}\right) \cdot \bar{t}\right)^{S} \sum_{d \geqslant 1}(-1)^{(d-1)(r+1)} d q^{d \ell}=-\left(c_{1}\left(F+F^{\prime}\right) \cdot \bar{t}\right)^{S} \delta_{h} \mathbf{f}\left(q^{\ell}\right) .
$$

This higher-order series does not occur as a correction to the classical defect, though it is still derived from the information on the case $\nu=0$ together with the classical (bundle-theoretic) data. Of course, it is invariant under $P^{r}$ flops in terms of analytic continuation.

Remark 3.3. It is helpful to comment on $\bar{t} h^{j}$ and $\mathscr{F}\left(\bar{t} h^{j}\right)$ to avoid confusion. Since the GromovWitten theory of extremal curve classes localizes to $Z$, the class $\bar{t} h^{j}$ is regarded as $\left.a\right|_{Z}$ for some $a \in H(X)$. If $j \leqslant r$, the familiar formula $\left.\mathscr{F} a\right|_{Z^{\prime}}=(-1)^{j} \bar{t} h^{\prime j}$ follows from Proposition 2.2, Lemma 2.4 and the invariance of the Poincaré pairing. However, this formula is not true for $j>r$. Instead, by the Segre relation $\bar{\psi}_{*} h^{r+\nu}=s_{\nu}$, we find $h^{r+\nu}=s_{\nu} h^{r}+$ (lower-order terms). This observation will be useful later.

\subsection{Twisted relative invariants for general $\nu$}

We will show that when $\sum_{i=1}^{3} j_{i}=2 r+1+\nu(\nu \leqslant r-1)$, there is a degree $\nu$ polynomial $W_{\nu}^{F, F^{\prime}}(d)=\sum_{i=0}^{\nu} w_{\nu, i}\left(F, F^{\prime}\right) d^{i}$ with coefficients $w_{\nu, i}\left(F, F^{\prime}\right) \in H^{2 \nu}(S, \mathbb{Q})$ such that for any class $\bar{t} \in H^{2(r-\nu)}(S)$,

$$
\begin{aligned}
\left\langle h^{j_{1}}, h^{j_{2}}, \bar{t} h^{j_{3}}\right\rangle_{d} & =(-1)^{(d-1)(r+1)}\left(W_{\nu}^{F, F^{\prime}} \cdot \bar{t}\right)^{S}(d) \\
& :=(-1)^{(d-1)(r+1)} \sum_{i=0}^{\nu}\left(w_{\nu, i}\left(F, F^{\prime}\right) \cdot \bar{t}\right)^{S} d^{i} .
\end{aligned}
$$

Hence the 3-point extremal correction is given by

$$
\left\langle h^{j_{1}}, h^{j_{2}}, \bar{t} h^{j_{3}}\right\rangle_{+}:=\sum_{d \geqslant 1}\left\langle h^{j_{1}}, h^{j_{2}}, \bar{t} h^{j_{3}}\right\rangle_{d} q^{d \ell}=\left(W_{\nu}^{F, F^{\prime}} \cdot \bar{t}\right)^{S}\left(\delta_{h}\right) \mathbf{f}\left(q^{\ell}\right),
$$

and the corresponding $\bar{\psi}$-relative invariant is equal to

$$
\left\langle h^{j_{1}}, h^{j_{2}}, h^{j_{3}}\right\rangle_{+}^{/ S}=W_{\nu}^{F, F^{\prime}}\left(\delta_{h}\right) \mathbf{f}\left(q^{\ell}\right) .
$$

The constant term of $W_{\nu}^{F, F^{\prime}}$ is the $\nu$ th Segre class of $F+F^{\prime *}$. This is what we need because (as in the $\nu=1$ case)

$$
\tilde{s}_{\nu} \mathbf{f}\left(q^{\ell}\right)-(-1)^{j_{1}+j_{2}+j_{3}} \tilde{s}_{\nu}^{\prime} \mathbf{f}\left(q^{\ell^{\prime}}\right)=(-1)^{r} \tilde{s}_{\nu}
$$

That is, the classical defect is corrected.

Similarly, for the $d^{i}$ component with $i \geqslant 1$,

$$
w_{\nu, i} \delta_{h}^{i} \mathbf{f}\left(q^{\ell}\right)=w_{\nu, i}\left(-\delta_{h^{\prime}}\right)^{i}\left((-1)^{r}-\mathbf{f}\left(q^{\ell^{\prime}}\right)\right)=(-1)^{i+1} w_{\nu, i} \delta_{h^{\prime}}^{i} \mathbf{f}\left(q^{\ell^{\prime}}\right) .
$$

This is expected to agree with $(-1)^{j_{1}+j_{2}+j_{3}} w_{\nu, i}^{\prime} \delta_{h^{\prime}}^{i} \mathbf{f}\left(q^{\ell^{\prime}}\right)$. Hence we require the alternating nature of $W$ :

$$
w_{\nu, i}\left(F^{\prime}, F\right)=(-1)^{\nu+i} w_{\nu, i}\left(F, F^{\prime}\right) .
$$


Remark 3.4. We ignore the degree zero (classical) invariants in the formulation since they depend on the global geometry of $X$ and $X^{\prime}$ and could not be expressed by a local universal formula (only their difference could be).

Recall that for $1 \leqslant \nu \leqslant r-1$, any 3 -point invariant $\left\langle\bar{t}_{1} h^{j_{1}}, \bar{t}_{2} h^{j_{2}}, \bar{t}_{3} h^{j_{3}}\right\rangle_{d}$ with $1 \leqslant j_{i} \leqslant r$ and $\sum j_{i}=(2 r+1)+\nu$ is equal to the standard form $\left\langle h^{\nu+1}, h^{r}, \bar{t} h^{r}\right\rangle_{d}$, where $\bar{t}=\bar{t}_{1} \bar{t}_{2} \bar{t}_{3} \in H^{2(s-\nu)}(S)$. Its study is based on the recursive formula on extremal corrections $W_{\nu}:=\left\langle h^{\nu+1}, h^{r}, h^{r}\right\rangle_{+}^{/ S}$.

Proposition 3.5. $W_{\nu}=s_{\nu} \mathbf{f}+\sum_{j=1}^{\nu} W_{\nu-j}\left((-1)^{r} c_{j} \mathbf{f}-(-1)^{r+j} c_{j}^{\prime} \mathbf{f}-c_{j}\right)$.

Proof. As in [LLW10], by using the operator $\delta_{h}$, the divisor relation can be used to obtain a splitting relation of generating series,

$$
\left\langle h^{\nu+1}, h^{r}, \bar{t} h^{r}\right\rangle_{+}=\left\langle h^{\nu}, h^{r+1}, \bar{t} h^{r}\right\rangle_{+}+\sum_{i}\left\langle h^{\nu}, \bar{t} h^{r}, T_{\mu}\right\rangle_{+} \delta_{h}\left\langle T^{\mu}, h^{r}\right\rangle_{+}+\left(s_{\nu} \cdot \bar{t}\right)^{S} \mathbf{f} .
$$

The last term comes from the case $d_{1}=0$ :

$$
\sum_{\mu}\left\langle h^{\nu}, \bar{t} h^{r}, T_{\mu}\right\rangle_{0} \delta_{h}\left\langle T^{\mu}, h^{r}\right\rangle_{+}=\delta_{h}\left\langle\bar{t} h^{\nu+r}, h^{r}\right\rangle_{+}=\left(s_{\nu} \cdot \bar{t}\right)^{S} \mathbf{f} .
$$

Here we use the Segre relation $h^{r+\nu}=s_{\nu} h^{r}+$ (lower-order terms) and our complete knowledge of 2-point invariants.

By the Chern polynomial relation, the first term of (3.3) equals

$$
-\sum_{j=1}^{\nu}\left\langle h^{\nu}, c_{j} h^{r+1-j}, \bar{t} h^{r}\right\rangle_{+}=-\sum_{j=1}^{\nu}\left\langle h^{\nu-j+1}, h^{r}, c_{j} \bar{t} h^{r}\right\rangle_{+}=-\sum_{j=1}^{\nu}\left(W_{\nu-j} \cdot c_{j} \bar{t}\right)^{S} .
$$

For the second term of (3.3), we take the degree $r+1$ part of the $T_{\mu}$, which are of the form $\left\{\bar{t}_{j} h^{r+1-j}\right\}_{j=1}^{\nu}$ with $\bar{t}_{j} \in H^{2 j}(S)$ to be determined later. Then, as in the previous calculation, using local models, the corresponding dual basis $\left\{T^{\mu}\right\}$ is given by $\left\{\check{\bar{t}}_{j} H_{j-1} \Theta_{r+1}\right\}_{j=1}^{\nu}$. We need the $h^{r}$ part of

$$
H_{j-1} \Theta_{r+1}=(-1)^{r+1}\left(h^{j-1}+c_{1} h^{j-2}+\cdots+c_{j-1}\right)\left(h^{r+1}-c_{1}^{\prime} h^{r}+\cdots+(-1)^{r+1} c_{r+1}^{\prime}\right)
$$

in the standard presentation of $H(Z)$. As $\tilde{c}:=c\left(F+F^{\prime *}\right)$ equals $c(F) c\left(F^{* *}\right)$, it is $(-1)^{r+1}$ times the $h^{r}$ part of

$$
h^{r}\left(\tilde{c}_{j}-c_{j}\right)+h^{r+1} \tilde{c}_{j-1}+h^{r+2} \tilde{c}_{j-2}+\cdots+h^{r+j} .
$$

By the Segre relation and $c\left(F^{\prime *}\right)=s(F) c\left(F+F^{\prime *}\right)$, the term is

$$
h^{r}\left(\tilde{c}_{j}+s_{1} \tilde{c}_{j-1}+s_{2} \tilde{c}_{j-2}+\cdots+s_{j-1} \tilde{c}_{1}+s_{j}-c_{j}\right)=h^{r}\left((-1)^{j} c_{j}^{\prime}-c_{j}\right) .
$$

Now, we let $\bar{t}_{j}=(-1)^{j} c_{j}^{\prime}-c_{j}$, and the sum becomes

$$
(-1)^{r+1} \sum_{j=1}^{\nu}\left\langle h^{\nu}, \bar{t} h^{r}, \bar{t}_{j} h^{r+1-j}\right\rangle_{+} \mathbf{f}=(-1)^{r+1} \sum_{j=1}^{\nu}\left(W_{\nu-j}\left((-1)^{j} c_{j}^{\prime}-c_{j}\right) \mathbf{f} \cdot \bar{t}\right)^{S} .
$$

The result follows by combining the expressions for the three terms of (3.3).

TheOREM 3.6. The $\bar{\psi}$-relative invariant over $S$

$$
W_{\nu}=\left\langle h^{j_{1}}, h^{j_{2}}, h^{j_{3}}\right\rangle_{+}^{/ S}
$$

with $1 \leqslant j_{i} \leqslant r$ and $\nu=\sum j_{i}-(2 r+1) \leqslant r-1$ is the action on $\mathbf{f}$ by a universal (in $c(F)$ and $\left.c\left(F^{\prime}\right)\right)$ polynomial of degree $\nu$ in $\delta_{h}$ with rational cohomology classes as coefficients, which 


\section{INVARIANCE OF QUANTUM RINGS I}

is independent of the choices of the $j_{i}$ and satisfies the functional equation

$$
W_{\nu}-(-1)^{\nu+1} W_{\nu}^{\prime}=(-1)^{r} \tilde{s}_{\nu}
$$

for $0 \leqslant \nu \leqslant r-1$.

Proof. Since $W_{0}=\mathbf{f}$, by Proposition 3.5, it is clear that $W_{\nu}$ is recursively and uniquely determined. It is a degree $\nu+1$ polynomial in $\mathbf{f}$ with coefficients that are universal polynomials in $c(F)$ and $c\left(F^{\prime}\right)$ of pure degree $\nu$.

Let

$$
\delta=\delta_{h}=q d / d q .
$$

In order to rewrite $W_{\nu}$ as a degree $\nu$ polynomial in $\delta \mathbf{f}$, we start with the basic relation

$$
\delta \mathbf{f}=\mathbf{f}+(-1)^{r+1} \mathbf{f}^{2} .
$$

Since $\delta(f g)=(\delta f) g+f \delta g$, it follows inductively that $\delta^{m} \mathbf{f}$ can be expressed as $P_{m}(\mathbf{f})=\mathbf{f}+$ $\cdots+(-1)^{m(r+1)} m ! \mathbf{f}^{m+1}$ with $P_{m}$ an integral universal polynomial of degree $m+1$. Solving the upper triangular system between $\delta^{m} \mathbf{f}$ and $\mathbf{f}^{m+1}$ gives $\mathbf{f}^{\nu+1}=(-1)^{m(r+1)} \delta^{\nu} \mathbf{f} / \nu !+\cdots=Q_{\nu}(\delta) \mathbf{f}$ with $Q_{\nu}$ a rational polynomial. Clearly $W_{\nu}$ then admits a corresponding expression with rational cohomology classes as coefficients, as expected.

It remains to check that $W_{\nu}$ satisfies the required functional equation

$$
W_{\nu}-(-1)^{\nu+1} W_{\nu}^{\prime}=(-1)^{r} \tilde{s}_{\nu}
$$

We will prove this by induction. The case $\nu=0$ goes back to $\mathbf{f}+\mathbf{f}^{\prime}=(-1)^{r}$, where $\mathbf{f}:=\mathbf{f}\left(q^{\ell}\right)$ and $\mathbf{f}^{\prime}:=\mathbf{f}\left(q^{\ell^{\prime}}\right) \equiv \mathbf{f}\left(q^{-\ell}\right)$ under the correspondence $\mathscr{F}$.

Assume that the functional equation holds for all $j<\nu$. Then

$$
\begin{aligned}
& W_{\nu}=s_{\nu} \mathbf{f}+\sum_{j=1}^{\nu} W_{\nu-j}\left((-1)^{r} c_{j} \mathbf{f}-(-1)^{r+j} c_{j}^{\prime} \mathbf{f}-c_{j}\right), \\
& W_{\nu}^{\prime}=s_{\nu}^{\prime} \mathbf{f}^{\prime}+\sum_{j=1}^{\nu} W_{\nu-j}^{\prime}\left((-1)^{r} c_{j}^{\prime} \mathbf{f}^{\prime}-(-1)^{r+j} c_{j} \mathbf{f}^{\prime}-c_{j}^{\prime}\right) .
\end{aligned}
$$

By substituting

$$
W_{\nu-j}^{\prime}=(-1)^{\nu-j+1} W_{\nu-j}+(-1)^{r+\nu-j} \tilde{s}_{\nu-j}
$$

into $W_{\nu}^{\prime}$, we compute, after cancellations,

$$
\begin{aligned}
W_{\nu}-(-1)^{\nu+1} W_{\nu}^{\prime} & =s_{\nu} \mathbf{f}+(-1)^{\nu} s_{\nu}^{\prime} \mathbf{f}^{\prime}+\sum_{j=1}^{\nu}\left((-1)^{j} \tilde{s}_{\nu-j} c_{j}^{\prime} \mathbf{f}^{\prime}-\tilde{s}_{\nu-j} c_{j} \mathbf{f}^{\prime}-(-1)^{r-j} \tilde{s}_{\nu-j} c_{j}^{\prime}\right) \\
& =s_{\nu} \mathbf{f}+(-1)^{\nu} s_{\nu}^{\prime} \mathbf{f}^{\prime}+\left(s_{\nu}-\tilde{s}_{\nu}\right) \mathbf{f}^{\prime}-\left((-1)^{\nu} s_{\nu}^{\prime}-\tilde{s}_{\nu}\right) \mathbf{f}^{\prime}-(-1)^{r}\left(s_{\nu}-\tilde{s}_{\nu}\right) \\
& =s_{\nu}\left(\mathbf{f}+\mathbf{f}^{\prime}\right)-(-1)^{r} s_{\nu}+(-1)^{r} \tilde{s}_{\nu} \\
& =(-1)^{r} \tilde{s}_{\nu},
\end{aligned}
$$

where both directions of the Whitney sum relations

$$
s(F)=s\left(F+F^{\prime *}\right) c\left(F^{*}\right), \quad s\left(F^{\prime *}\right)=s\left(F+F^{\prime *}\right) c(F)
$$

are used. The proof is complete.

COROllary 3.7. For any ordinary flop over a smooth base, we have

$$
\mathscr{F}\left\langle a_{1}, a_{2}, a_{3}\right\rangle^{X} \cong\left\langle\mathscr{F} a_{1}, \mathscr{F} a_{2}, \mathscr{F} a_{3}\right\rangle^{X^{\prime}}
$$


modulo non-extremal curve classes.

\subsection{Functional equations for $n$-point extremal functions with $n \geqslant 3$}

For ordinary flops over any smooth base, we will show that Corollary 3.7 extends to all $n \geqslant 4$. Namely,

$$
\mathscr{F}\left\langle a_{1}, \ldots, a_{n}\right\rangle^{X} \cong\left\langle\mathscr{F} a_{1}, \ldots, \mathscr{F} a_{n}\right\rangle^{X^{\prime}}
$$

modulo non-extremal curve classes.

By restricting to $Z$ and $Z^{\prime}$, this is equivalent to the nice-looking formula

$$
\mathscr{F}\left\langle h^{j_{1}}, \ldots, \bar{t} h^{j_{n}}\right\rangle \cong(-1)^{\sum j_{i}}\left\langle h^{\prime j_{1}}, \ldots, \bar{t} h^{\prime j_{n}}\right\rangle
$$

for all $1 \leqslant j_{l} \leqslant r$, where for notational simplicity the $n$-point functions in this section refer to extremal functions, that is, the sum is only over $\mathbb{Z}_{+} \ell$.

Notice that $\mathscr{F}\left(\bar{t} h^{j}\right)=(-1)^{j} \bar{t} h^{\prime j}$ holds only for $j \leqslant r$ and fails in general for $j>r$ if the base $S$ is non-trivial. In fact, the following holds.

Lemma 3.8. We have $\mathscr{F}\left(h^{r+1}\right)-(\mathscr{F} h)^{r+1}=(-1)^{r+1} \mathscr{F} \Theta_{r+1}$ along $Z^{\prime}$.

Proof. This is simply a reformulation of Lemma 3.1.

It is easy to see that $\mathscr{F}\left\langle h^{j_{1}}, \ldots, \bar{t} h^{j_{n}}\right\rangle \not(-1)^{\sum j_{i}}\left\langle h^{\prime j_{1}}, \ldots, \bar{t} h^{\prime j_{n}}\right\rangle$ if $j_{l}>r$ for some $l$. This appears as the subtle point in proving the functional equations for $n \geqslant 4$ points. The above lemma plays a crucial role in analyzing this.

Theorem 3.9. Let $f: X \rightarrow X^{\prime}$ be an ordinary $P^{r}$ flop with exceptional loci $Z=P(F) \rightarrow S$ and $Z^{\prime}=P\left(F^{\prime}\right) \rightarrow S$. Then for $n \geqslant 3$,

$$
\mathscr{F}\left\langle h^{j_{1}}, \ldots, \bar{t} h^{j_{n}}\right\rangle^{X} \cong\left\langle\mathscr{F} h^{j_{1}}, \ldots, \mathscr{F} \bar{t} h^{j_{n}}\right\rangle^{X^{\prime}}
$$

for all $j_{l}$ and $\bar{t} \in H^{2(s-\nu)}(S)$ with $\nu=\sum_{l=1}^{n} j_{l}-(2 r+1+n-3)$.

Proof. This holds for $n=3$ by Corollary 3.7. Suppose that this has been proven up to some $n \geqslant 3$. The basic idea is that an iterated application of the divisor relation using the operator $\delta_{h}$ should allow us to reduce an $(n+1)$-point extremal function to one with fewer marked points. The technical details, however, should be traced carefully.

The first point to make is on the diagonal splitting $\Delta(X)=\sum T_{\mu} \otimes T^{\mu}$. Since the Poincaré pairing is preserved, $\mathscr{F} T^{\mu}$ is still the dual basis of $\mathscr{F} T_{\mu}$ in $H\left(X^{\prime}\right)$. Thus we may take the diagonal splitting on the $X^{\prime}$ side to be $\Delta\left(X^{\prime}\right)=\sum \mathscr{F} T_{\mu} \otimes \mathscr{F} T^{\mu}$.

We only need to prove the case where $j_{l} \leqslant r$ for all $l$. The $P^{1}$ flops always have $\nu=0$ and the proof is reduced to the simple case. So we assume $r \geqslant 2$.

We will prove the functional equation by further induction on $j_{1}$. The case $j_{1}=1$ holds by the divisor axiom and induction, so we assume $j_{1} \geqslant 2$. By applying the divisor relation to $(i, j, k)=(1,2,3)$, we get

$$
\begin{aligned}
\left\langle h^{j_{1}}, h^{j_{2}}, h^{j_{3}}, \ldots\right\rangle= & \left\langle h^{j_{1}-1}, h^{j_{2}+1}, h^{j_{3}}, \ldots\right\rangle+\sum_{\mu}\left\langle h^{j_{1}-1}, h^{j_{3}}, \ldots, T_{\mu}\right\rangle \delta_{h}\left\langle h^{j_{2}}, \ldots, T^{\mu}\right\rangle \\
& -\delta_{h}\left\langle h^{j_{1}-1}, \ldots, T_{\mu}\right\rangle\left\langle h^{j_{2}}, h^{j_{3}}, \ldots, T^{\mu}\right\rangle .
\end{aligned}
$$

Since $j_{1}-1<r$, the term $\left\langle h^{j_{1}-1}, \ldots, T_{\mu}\right\rangle$ cannot be a 2 -point invariant unless it is trivial. Hence we may assume that $\left\langle h^{j_{2}}, h^{j_{3}}, \ldots, T^{\mu}\right\rangle$ has fewer marked points. 


\section{INVARIANCE OF QUANTUM RINGS I}

The term $\left\langle h^{j_{1}-1}, h^{j_{2}+1}, h^{j_{3}}, \ldots\right\rangle$ is also handled by induction since $j_{1}-1<j_{1}$. Thus we may apply $\mathscr{F}$ to the equation and apply induction to get

$$
\begin{aligned}
\mathscr{F}\left\langle h^{j_{1}}, h^{j_{2}}, h^{j_{3}}, \ldots\right\rangle= & \left\langle\mathscr{F} h^{j_{1}-1}, \mathscr{F} h^{j_{2}+1}, \mathscr{F} h^{j_{3}}, \ldots\right\rangle \\
& +\sum_{\mu}\left\langle\mathscr{F} h^{j_{1}-1}, \mathscr{F} h^{j_{3}}, \ldots, \mathscr{F} T_{\mu}\right\rangle \delta_{\mathscr{F} h} \mathscr{F}\left\langle h^{j_{2}}, \ldots, T^{\mu}\right\rangle \\
& -\delta_{\mathscr{F} h}\left\langle\mathscr{F} h^{j_{1}-1}, \ldots, \mathscr{F} T_{\mu}\right\rangle\left\langle\mathscr{F} h^{j_{2}}, \mathscr{F} h^{j_{3}}, \ldots, \mathscr{F} T^{\mu}\right\rangle,
\end{aligned}
$$

where $\mathscr{F} \circ \delta_{h}=\delta_{\mathscr{F} h} \circ \mathscr{F}$ by [LLW10, Lemma 5.5].

Notice that in the sum, we have

$$
\mathscr{F}\left\langle h^{j_{2}}, \ldots, T^{\mu}\right\rangle=\left\langle\mathscr{F} h^{j_{2}}, \ldots, \mathscr{F} T^{\mu}\right\rangle
$$

if it is not a 2-point invariant. Also, the 2-point case survives precisely when $j_{2}=r$ and $T^{\mu}=$ $\mathrm{pt} \cdot h^{r}$. In that case, by the invariance of 3-point extremal functions in the $\nu=0$ (simple) case, the corresponding term becomes

$$
\mathscr{F} \delta_{h}\left\langle h^{r}, T^{\mu}\right\rangle=\mathscr{F}\left\langle h, h^{r}, T^{\mu}\right\rangle_{+}=\left\langle\mathscr{F} h, \mathscr{F} h^{r}, \mathscr{F} T^{\mu}\right\rangle_{+}+(-1)^{r}=\delta_{\mathscr{F} h}\left\langle\mathscr{F} h^{r}, \mathscr{F} T^{\mu}\right\rangle+(-1)^{r} .
$$

We also have $\left.T_{\mu}\right|_{Z}=\left.\Theta_{r+1}\right|_{Z}$. Hence, by Lemma 3.8 the extra $(-1)^{r}$ contributes

$$
-\left\langle\mathscr{F} h^{j_{1}-1}, \mathscr{F} h^{j_{3}}, \ldots, \mathscr{F} h^{r+1}\right\rangle-\left\langle\mathscr{F} h^{j_{1}-1}, \mathscr{F} h^{j_{3}}, \ldots,(\mathscr{F} h)^{r+1}\right\rangle .
$$

Since $j_{2}=r$, the first term cancels out with the first term in the divisor relation (3.4) and we end up with the second term as the main term of (3.4).

Now, we compare (3.4) with the similar divisor relation for

$$
\left\langle\mathscr{F} h^{j_{1}}, \mathscr{F} h^{j_{2}}, \mathscr{F} h^{j_{3}}, \ldots\right\rangle=\left\langle\mathscr{F} h \cdot \mathscr{F} h^{j_{1}-1}, \mathscr{F} h^{j_{2}}, \mathscr{F} h^{j_{3}}, \ldots\right\rangle
$$

under the diagonal splitting $\Delta\left(X^{\prime}\right)=\sum_{\mu} \mathscr{F} T_{\mu} \otimes \mathscr{F} T^{\mu}$. Namely,

$$
\begin{aligned}
\left\langle\mathscr{F} h^{j_{1}}, \mathscr{F} h^{j_{2}}, \mathscr{F} h^{j_{3}}, \ldots\right\rangle= & \left\langle\mathscr{F} h^{j_{1}-1}, \mathscr{F} h . \mathscr{F} h^{j_{2}}, \mathscr{F} h^{j_{3}}, \ldots\right\rangle \\
& +\sum_{\mu}\left\langle\mathscr{F} h^{j_{1}-1}, \mathscr{F} h^{j_{3}}, \ldots, \mathscr{F} T_{\mu}\right\rangle \delta \mathscr{F} h\left\langle\mathscr{F} h^{j_{2}}, \ldots, \mathscr{F} T^{\mu}\right\rangle \\
& -\delta_{\mathscr{F} h}\left\langle\mathscr{F} h^{j_{1}-1}, \ldots, \mathscr{F} T_{\mu}\right\rangle\left\langle\mathscr{F} h^{j_{2}}, \mathscr{F} h^{j_{3}}, \ldots, \mathscr{F} T^{\mu}\right\rangle .
\end{aligned}
$$

If $j_{2}<r$, then there is no 2-point splitting and $\mathscr{F} h \cdot \mathscr{F} h^{j_{2}}=\mathscr{F} h^{j_{2}+1}$, hence the functional equation holds. If $j_{2}=r$, then $\mathscr{F} h \cdot \mathscr{F} h^{r}=(\mathscr{F} h)^{r+1}$. This again agrees with the main term of (3.4) obtained above. Hence the proof of the functional equations is complete by induction.

The formula for $W_{\vec{j}}:=\left\langle h^{j_{1}}, \ldots, h^{j_{n}}\right\rangle^{/ S}$ can be achieved by a process similar to that in the proof of Proposition 3.5. Its exact form will not be pursued here. In general it depends on the vector $\vec{j}$ instead of $\sum j_{i}$.

Remark 3.10. Theorems 3.6 and 3.9 (for the special case $F^{\prime}=F^{*}$ ) have been applied in [FW08] to study stratified Mukai flops. In particular, they provide non-trivial quantum corrections to flops of type $A_{n, 2}, D_{5}$ and $E_{6, I}$.

\section{Degeneration analysis revisited}

Our next task is to compare the Gromov-Witten invariants of $X$ and $X^{\prime}$ for all genera and for curve classes other than the flopped curve. As in [LLW10], we use the degeneration formula [LR01, Li02] to reduce the problem to local models. This has been achieved for simple ordinary 
flops in [LLW10] for genus zero invariants. In this section we extend the argument to the general case and establish Theorem 1.1 (as the combination of Propositions 4.3 and 4.7).

\subsection{The degeneration formula}

We start by reviewing the basic set-up. Details can be found in the above references.

Consider a pair $(Y, E)$ with $E \hookrightarrow Y$ a smooth divisor. Let $\Gamma=(g, n, \beta, \rho, \mu)$ with $\mu=$ $\left(\mu_{1}, \ldots, \mu_{\rho}\right) \in \mathbb{N}^{\rho}$ a partition of the intersection number $(\beta \cdot E)=|\mu|:=\sum_{i=1}^{\rho} \mu_{i}$. For $A \in H(Y)^{\otimes n}$ and $\varepsilon \in H(E)^{\otimes \rho}$, the relative invariant of stable maps with topological type $\Gamma$ (that is, with contact order $\mu_{i}$ in $E$ at the $i$ th contact point) is

$$
\langle A \mid \varepsilon, \mu\rangle_{\Gamma}^{(Y, E)}:=\int_{\left[\bar{M}_{\Gamma}(Y, E)\right]^{\mathrm{virt}}} e_{Y}^{*} A \cup e_{E}^{*} \varepsilon,
$$

where $e_{Y}: \bar{M}_{\Gamma}(Y, E) \rightarrow Y^{n}$ and $e_{E}: \bar{M}_{\Gamma}(Y, E) \rightarrow E^{\rho}$ are evaluation maps on marked points and contact points, respectively. If $\Gamma=\coprod_{\pi} \Gamma^{\pi}$, the relative invariant with disconnected domain curve is defined by the product rule:

$$
\langle A \mid \varepsilon, \mu\rangle_{\Gamma}^{\bullet(Y, E)}:=\prod_{\pi}\langle A \mid \varepsilon, \mu\rangle_{\Gamma^{\pi}}^{(Y, E)}
$$

We apply the degeneration formula to the following situation. Let $X$ be a smooth variety and $Z \subset X$ a smooth subvariety. Let $\Phi: W \rightarrow \mathscr{X}$ be its degeneration to the normal cone, the blow-up of $X \times \mathbb{A}^{1}$ along $Z \times\{0\}$. Let $t \in \mathbb{A}^{1}$. Then $W_{t} \cong X$ for all $t \neq 0$ and $W_{0}=Y_{1} \cup Y_{2}$ with

$$
\phi=\left.\Phi\right|_{Y_{1}}: Y_{1} \rightarrow X
$$

the blow-up along $Z$ and

$$
p=\left.\Phi\right|_{Y_{2}}: Y_{2}:=P\left(N_{Z / X} \oplus \mathscr{O}\right) \rightarrow Z \subset X
$$

the projective completion of the normal bundle. The intersection $Y_{1} \cap Y_{2}=: E=P\left(N_{Z / X}\right)$ is the $\phi$-exceptional divisor which consists of the infinity part.

The family $W \rightarrow \mathbb{A}^{1}$ is a degeneration of a trivial family, so all cohomology classes $\alpha \in$ $H(X, \mathbb{Z})^{\oplus n}$ have global liftings and the restriction $\alpha(t)$ on $W_{t}$ is defined for all $t$. Let $j_{i}: Y_{i} \hookrightarrow W_{0}$ be the inclusion maps for $i=1,2$. Let $\left\{\mathbf{e}_{i}\right\}$ be a basis of $H(E)$, with $\left\{\mathbf{e}^{i}\right\}$ its dual basis. Then $\left\{\mathbf{e}_{I}\right\}$ forms a basis of $H\left(E^{\rho}\right)$ with dual basis $\left\{\mathbf{e}^{I}\right\}$, where $|I|=\rho$ and $\mathbf{e}_{I}=\mathbf{e}_{i_{1}} \otimes \cdots \otimes \mathbf{e}_{i_{\rho}}$. The degeneration formula expresses the absolute invariants of $X$ in terms of the relative invariants of the two smooth pairs $\left(Y_{1}, E\right)$ and $\left(Y_{2}, E\right)$ :

$$
\langle\alpha\rangle_{g, n, \beta}^{X}=\sum_{I} \sum_{\eta \in \Omega_{\beta}} C_{\eta}\left\langle j_{1}^{*} \alpha(0) \mid \mathbf{e}_{I}, \mu\right\rangle_{\Gamma_{1}}^{\bullet\left(Y_{1}, E\right)}\left\langle j_{2}^{*} \alpha(0) \mid \mathbf{e}^{I}, \mu\right\rangle_{\Gamma_{2}}^{\bullet\left(Y_{2}, E\right)} .
$$

Here $\eta=\left(\Gamma_{1}, \Gamma_{2}, I_{\rho}\right)$ is an admissible triple which consists of (possibly disconnected) topological types

$$
\Gamma_{i}=\coprod_{\pi=1}^{\left|\Gamma_{i}\right|} \Gamma_{i}^{\pi}
$$

with the same partition $\mu$ of the contact order under the identification $I_{\rho}$ of the contact points. The gluing $\Gamma_{1}+{ }_{I_{\rho}} \Gamma_{2}$ has type $(g, n, \beta)$ and is connected. In particular, $\rho=0$ if and only if one of the $\Gamma_{i}$ is empty. The total genus $g_{i}$, the total number of marked points $n_{i}$ and the total degree 


\section{INVARIANCE OF QUANTUM RINGS I}

$\beta_{i} \in \mathrm{NE}\left(Y_{i}\right)$ satisfy the splitting relations

$$
\begin{aligned}
g-1 & =\sum_{\pi=1}^{\left|\Gamma_{1}\right|}\left(g_{1}(\pi)-1\right)+\sum_{\pi=1}^{\left|\Gamma_{2}\right|}\left(g_{2}(\pi)-1\right)+\rho \\
& =g_{1}+g_{2}-\left|\Gamma_{1}\right|-\left|\Gamma_{2}\right|+\rho, \\
n & =n_{1}+n_{2}, \\
\beta & =\phi_{*} \beta_{1}+p_{*} \beta_{2} .
\end{aligned}
$$

(The first one is the arithmetic genus relation for nodal curves.)

Moreover, $C_{\eta}=m(\mu) / \mid$ Aut $\eta \mid$, where $m(\mu)=\prod \mu_{i}$ and Aut $\eta=\left\{\sigma \in S_{\rho} \mid \eta^{\sigma}=\eta\right\}$. We denote by $\Omega$ the set of equivalence classes of all admissible triples, and by $\Omega_{\beta}$ and $\Omega_{\mu}$ the subsets with fixed degree $\beta$ and fixed contact order $\mu$, respectively.

Given an ordinary flop $f: X \rightarrow X^{\prime}$, we apply degeneration to the normal cone to both $X$ and $X^{\prime}$. Then $Y_{1} \cong Y_{1}^{\prime}$ and $E=E^{\prime}$ by the definition of ordinary flops. The following notation will be used:

$$
Y:=\mathrm{Bl}_{Z} X \cong Y_{1} \cong Y_{1}^{\prime}, \quad \tilde{E}:=P\left(N_{Z / X} \oplus \mathscr{O}\right), \quad \tilde{E}^{\prime}:=P\left(N_{Z^{\prime} / X^{\prime}} \oplus \mathscr{O}\right)
$$

Next, we discuss the presentation of $\alpha(0)$. Denote by $\iota_{1} \equiv j: E \hookrightarrow Y_{1}=Y$ and $\iota_{2}: E \hookrightarrow Y_{2}=$ $\tilde{E}$ the natural inclusions. The class $\alpha(0)$ can be represented by $\left(j_{1}^{*} \alpha(0), j_{2}^{*} \alpha(0)\right)=\left(\alpha_{1}, \alpha_{2}\right)$ with $\alpha_{i} \in H\left(Y_{i}\right)$ such that

$$
\iota_{1}^{*} \alpha_{1}=\iota_{2}^{*} \alpha_{2} \quad \text { and } \quad \phi_{*} \alpha_{1}+p_{*} \alpha_{2}=\alpha .
$$

Such representatives are called liftings; they are not unique.

The standard choice of lifting is

$$
\alpha_{1}=\phi^{*} \alpha \quad \text { and } \quad \alpha_{2}=p^{*}\left(\left.\alpha\right|_{Z}\right) .
$$

Other liftings can be obtained from the standard one in the following way.

Lemma 4.1 ([LLW10]). Let $\alpha(0)=\left(\alpha_{1}, \alpha_{2}\right)$ be a choice of lifting. Then

$$
\alpha(0)=\left(\alpha_{1}-\iota_{1 *} e, \alpha_{2}+\iota_{2 *} e\right)
$$

is also a lifting for any class $e$ in $E$ of the same dimension as $\alpha$. Moreover, any two liftings are related in this manner.

For an ordinary flop $f: X \rightarrow X^{\prime}$, we compare the degeneration expressions of $X$ and $X^{\prime}$. For a given admissible triple $\eta=\left(\Gamma_{1}, \Gamma_{2}, I_{\rho}\right)$ on the degeneration of $X$, one may pick the corresponding $\eta^{\prime}=\left(\Gamma_{1}^{\prime}, \Gamma_{2}^{\prime}, I_{\rho}^{\prime}\right)$ on the degeneration of $X^{\prime}$ in such a way that $\Gamma_{1}=\Gamma_{1}^{\prime}$. Since

$$
\phi^{*} \alpha-\phi^{*} \mathscr{F} \alpha \in \iota_{1 *} H(E) \subset H(Y),
$$

Lemma 4.1 implies that one can choose $\alpha_{1}=\alpha_{1}^{\prime}$. This procedure identifies relative invariants on $Y_{1}=Y=Y_{1}^{\prime}$ from both sides, and we are left with the comparison of the corresponding relative invariants on $\tilde{E}$ and $\tilde{E}^{\prime}$.

The ordinary flop $f$ induces an ordinary flop

$$
\tilde{f}: \tilde{E} \rightarrow \tilde{E}^{\prime}
$$

on the local model. Denote again by $\mathscr{F}$ the cohomology correspondence induced by the graph closure. 
Lemma 4.2 ([LLW10]). Let $f: X \rightarrow X^{\prime}$ be an ordinary flop. Consider $\alpha \in H(X)$ with liftings $\alpha(0)=\left(\alpha_{1}, \alpha_{2}\right)$ and $\mathscr{F} \alpha(0)=\left(\alpha_{1}^{\prime}, \alpha_{2}^{\prime}\right)$. Then

$$
\alpha_{1}=\alpha_{1}^{\prime} \quad \Longleftrightarrow \mathscr{F} \alpha_{2}=\alpha_{2}^{\prime}
$$

Now, we are in a position to apply the degeneration formula to reduce the problem to relative invariants of local models.

Notice that $A_{1}(\tilde{E})=\iota_{2 *} A_{1}(E)$, since both are projective bundles over $Z$. We then have

$$
\phi^{*} \beta=\beta_{1}+\beta_{2}
$$

by regarding $\beta_{2}$ as a class in $E \subset Y$ (cf. [LLW10]).

Define the generating series for genus $g$ (connected) invariants

$$
\langle A \mid \varepsilon, \mu\rangle_{g}^{(\tilde{E}, E)}:=\sum_{\beta_{2} \in \operatorname{NE}(\tilde{E})} \frac{1}{|\operatorname{Aut} \mu|}\langle A \mid \varepsilon, \mu\rangle_{g, \beta_{2}}^{(\tilde{E}, E)} q^{\beta_{2}}
$$

and the similar one with possibly disconnected domain curves

$$
\langle A \mid \varepsilon, \mu\rangle^{\bullet(\tilde{E}, E)}:=\sum_{\Gamma ; \mu_{\Gamma}=\mu} \frac{1}{|\operatorname{Aut} \Gamma|}\langle A \mid \varepsilon, \mu\rangle_{\Gamma}^{\bullet(\tilde{E}, E)} q^{\beta^{\Gamma}} \kappa^{g^{\Gamma}-|\Gamma|} .
$$

For connected invariants of genus $g$ we assign the $\kappa$-weight $\kappa^{g-1}$, while for disconnected ones we simply assign the product weights.

Proposition 4.3. To prove $\mathscr{F}\langle\alpha\rangle_{g}^{X} \cong\langle\mathscr{F} \alpha\rangle_{g}^{X^{\prime}}$ for all $\alpha$ up to genus $g \leqslant g_{0}$, it is enough to show

$$
\mathscr{F}\langle A \mid \varepsilon, \mu\rangle_{g}^{(\tilde{E}, E)} \cong\langle\mathscr{F} A \mid \varepsilon, \mu\rangle_{g}^{\left(\tilde{E}^{\prime}, E\right)}
$$

for all $A, \varepsilon, \mu$ and $g \leqslant g_{0}$.

Proof. For the $n$-point function

$$
\langle\alpha\rangle^{X}=\sum_{g}\langle\alpha\rangle_{g}^{X} \kappa^{g-1}=\sum_{g ; \beta \in \operatorname{NE}(X)}\langle\alpha\rangle_{g, \beta}^{X} q^{\beta} \kappa^{g-1},
$$

the degeneration formula gives

$$
\begin{aligned}
& \langle\alpha\rangle^{X}=\sum_{g ; \beta \in \mathrm{NE}(X)} \sum_{\eta \in \Omega_{\beta}} \sum_{I} C_{\eta}\left\langle\alpha_{1} \mid \mathbf{e}_{I}, \mu\right\rangle_{\Gamma_{1}}^{\bullet\left(Y_{1}, E\right)}\left\langle\alpha_{2} \mid \mathbf{e}^{I}, \mu\right\rangle_{\Gamma_{2}}^{\bullet\left(Y_{2}, E\right)} q^{\phi^{*} \beta} \kappa^{g-1} \\
& =\sum_{\mu} \sum_{I} \sum_{\eta \in \Omega_{\mu}} C_{\eta}\left(\left\langle\alpha_{1} \mid \mathbf{e}_{I}, \mu\right\rangle_{\Gamma_{1}}^{\bullet\left(Y_{1}, E\right)} q^{\beta_{1}} \kappa^{g^{\Gamma_{1}}-\left|\Gamma_{1}\right|}\right)\left(\left\langle\alpha_{2} \mid \mathbf{e}^{I}, \mu\right\rangle_{\Gamma_{2}}^{\bullet\left(Y_{2}, E\right)} q^{\beta_{2}} \kappa^{g^{\Gamma_{2}-\left|\Gamma_{2}\right|}}\right) \kappa^{\rho} .
\end{aligned}
$$

(Notice that $\rho$ is determined by $\mu$.) In this formula, the variables $q^{\beta_{1}}$ on $Y_{1}$ and $q^{\beta_{2}}$ on $Y_{2}$ are identified with $q^{\phi_{*} \beta_{1}}$ and $q^{p_{*} \beta_{2}}$, respectively, on $X$.

To simplify the generating series, we also consider absolute invariants $\langle\alpha\rangle^{\bullet X}$ with possibly disconnected domain curves as in the relative case (with product weights in $\kappa$ ). Then, by comparing the order of the automorphisms, we deduce

$$
\langle\alpha\rangle^{\bullet X}=\sum_{\mu} m(\mu) \sum_{I}\left\langle\alpha_{1} \mid \mathbf{e}_{I}, \mu\right\rangle^{\bullet\left(Y_{1}, E\right)}\left\langle\alpha_{2} \mid \mathbf{e}^{I}, \mu\right\rangle^{\bullet\left(Y_{2}, E\right)} \kappa^{\rho} .
$$

To compare $\mathscr{F}\langle\alpha\rangle^{\bullet X}$ and $\langle\mathscr{F} \alpha\rangle^{\bullet X^{\prime}}$, by Lemma 4.2 we may assume $\alpha_{1}=\alpha_{1}^{\prime}$ and $\alpha_{2}^{\prime}=\mathscr{F} \alpha_{2}$. This choice of cohomology liftings identifies the relative invariants of $\left(Y_{1}, E\right)$ and those of $\left(Y_{1}^{\prime}, E\right)$ 


\section{INVARIANCE OF QUANTUM RINGS I}

with the same topological types. It remains to compare (cf. Remark 4.4 below)

$$
\left\langle\alpha_{2} \mid \mathbf{e}^{I}, \mu\right\rangle^{\bullet(\tilde{E}, E)} \text { and }\left\langle\mathscr{F} \alpha_{2} \mid \mathbf{e}^{I}, \mu\right\rangle^{\bullet\left(\tilde{E}^{\prime}, E\right)} \text {. }
$$

We further split the sum into connected invariants. Let $\Gamma^{\pi}$ be a connected part with contact order $\mu^{\pi}$ induced from $\mu$. Denote by $P: \mu=\sum_{\pi \in P} \mu^{\pi}$ a partition of $\mu$ and by $P(\mu)$ the set of all such partitions. Then

$$
\langle A \mid \varepsilon, \mu\rangle^{\bullet(\tilde{E}, E)}=\sum_{P \in P(\mu)} \prod_{\pi \in P} \sum_{\Gamma^{\pi}} \frac{1}{\left|\operatorname{Aut} \mu^{\pi}\right|}\left\langle A^{\pi} \mid \varepsilon^{\pi}, \mu^{\pi}\right\rangle_{\Gamma^{\pi}}^{(\tilde{E}, E)} q^{\beta^{\Gamma^{\pi}}} \kappa^{g^{\Gamma^{\pi}}-1} .
$$

In the summation over $\Gamma^{\pi}$, the indices to be summed over are $\beta^{\Gamma^{\pi}}$ on $\tilde{E}$ and the genus. This reduces the problem to $\left\langle A^{\pi} \mid \varepsilon^{\pi}, \mu^{\pi}\right\rangle_{g}^{(\tilde{E}, E)}$.

Instead of working with all genera, the proposition follows from the same argument by reduction modulo $\kappa^{g_{0}}$.

Remark 4.4. Notice that there is a natural compatibility on our identification of the curve classes which keeps track of the contact weight $|\mu|$. Namely, the identity

$$
\left\langle\alpha_{1} \mid \mathbf{e}_{I}, \mu\right\rangle^{\bullet\left(Y_{1}, E\right)}=\left\langle\alpha_{1} \mid \mathbf{e}_{I}, \mu\right\rangle^{\bullet\left(Y_{1}^{\prime}, E\right)}
$$

leads to

$$
\mathscr{F} \phi_{*}\left\langle\alpha_{1} \mid \mathbf{e}_{I}, \mu\right\rangle^{\bullet\left(Y_{1}, E\right)}=q^{|\mu| \ell^{\prime}} \phi_{*}^{\prime}\left\langle\alpha_{1} \mid \mathbf{e}_{I}, \mu\right\rangle^{\bullet\left(Y_{1}^{\prime}, E\right)},
$$

while $\mathscr{F}\left\langle\alpha_{2} \mid \mathbf{e}^{I}, \mu\right\rangle^{\bullet}(\tilde{E}, E) \cong\left\langle\mathscr{F} \alpha_{2} \mid \mathbf{e}^{I}, \mu\right\rangle^{\bullet\left(\tilde{E}^{\prime}, E\right)}$ leads to

$$
\mathscr{F} p_{*}\left\langle\alpha_{2} \mid \mathbf{e}^{I}, \mu\right\rangle^{\bullet(\tilde{E}, E)} \cong q^{-|\mu| \ell^{\prime}} p_{*}^{\prime}\left\langle\mathscr{F} \alpha_{2} \mid \mathbf{e}^{I}, \mu\right\rangle^{\bullet\left(\tilde{E}^{\prime}, E\right)} .
$$

Thus we may ignore the issue of contact weights in our discussion.

\subsection{From relative local back to absolute local}

Now, let $X=\tilde{E}$. We shall further reduce the relative cases to absolute cases with at most descendent insertions along $E$. This has been done in [LLW10] for genus zero invariants under simple flops. Here we extend the argument to ordinary flops over any smooth base $S$ and to all genera.

The local model

$$
\bar{p}:=\bar{\psi} \circ p: \tilde{E} \stackrel{p}{\rightarrow} Z \stackrel{\bar{\psi}}{\rightarrow} S,
$$

as well as the flop $f: \tilde{E} \rightarrow-\tilde{E}^{\prime}$, are defined over $S$, with each fiber isomorphic to the simple case. Thus the map on numerical 1-cycles

$$
\bar{p}_{*}: N_{1}(\tilde{E}) \rightarrow N_{1}(S)
$$

has kernel spanned by the $p$-fiber line class $\gamma$ and $\bar{\psi}$-fiber line class $\ell$, which is the flopping log-extremal ray.

Notice that for general $S$ the structure of $\mathrm{NE}(Z)$ could be complicated and $\mathrm{NE}(\tilde{E})$ is in general larger than $i_{*} \mathrm{NE}(Z) \oplus \mathbb{Z}^{+} \gamma$. For $\beta=\beta_{Z}+d_{2}(\beta) \gamma \in \mathrm{NE}(\tilde{E})$, while $\beta_{Z}=p_{*} \beta$ is necessarily effective, $d_{2}(\beta)$ could possibly be negative if (and only if) $\beta_{Z} \neq 0$. Nevertheless, we have the following. 
Lemma 4.5. The correspondence $\mathscr{F}$ is compatible with $N_{1}(S)$. Namely,

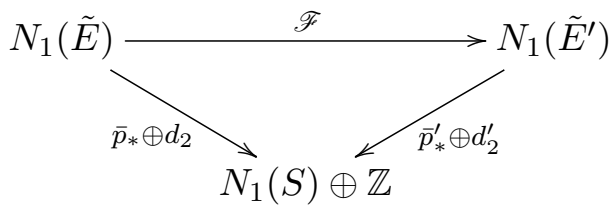

is commutative.

Proof. Since $N_{1}(\tilde{E})=i_{*} N_{1}(Z) \oplus \mathbb{Z} \gamma$ and $\mathscr{F} \gamma=\gamma^{\prime}+\ell^{\prime}$, we see that $d_{2}=d_{2}^{\prime} \circ \mathscr{F}$ and it is enough to consider $\beta \in N_{1}(Z)$. We also have $\mathscr{F} \ell=-\ell^{\prime}$, so the remaining cases are of the form $\beta=\bar{\psi}^{*} \beta_{S} \cdot H_{r}$ for $\beta_{S} \in N_{1}(S)$. Then $\mathscr{F} \beta=\bar{\psi}^{\prime *} \beta_{S} \cdot H_{r}^{\prime}$ and it is clear that both $\beta$ and $\mathscr{F} \beta$ project to $\beta_{S}$.

This leads to the following key observation, which applies to both absolute and relative invariants.

Proposition 4.6. The functional equation of a generating series $\langle A\rangle$ over the Mori cone of a local model $f: \tilde{E} \rightarrow \tilde{E}^{\prime}$ is equivalent to the functional equations of its various subseries (fiber series) $\langle A\rangle_{\beta_{S}, d_{2}}$ labeled by $\operatorname{NE}(S) \oplus \mathbb{Z}$. The fiber series is a sum over the affine rays $\beta \in\left(d_{2} \gamma+\bar{\psi}^{*} \beta_{S} \cdot H_{r}+\mathbb{Z} \ell\right) \cap \mathrm{NE}(\tilde{E})$.

To analyze these fiber series $\langle A\rangle_{\beta_{S}, d_{2}}$ with $\left(\beta_{S}, d_{2}\right) \in \mathrm{NE}(S) \oplus \mathbb{Z}$, we consider the partial order of effectivity (weight) of the quotient Mori cone

$$
W:=\mathrm{NE}(\tilde{E}) / \sim, \quad a \sim b \text { if and only if } a-b \in \mathbb{Z} \ell .
$$

Notice that $a>b$ and $b>a$ lead to $a \sim b$ since $\ell$ is an extremal ray. Under the natural identification, $W$ can be regarded as a subset of $\operatorname{NE}(S) \oplus \mathbb{Z}$. This is not the lexicographical (partial) order on $\operatorname{NE}(S) \oplus \mathbb{Z}$, though both notions are used in our discussions. For ease of notation we also use

$$
[\beta] \equiv\left(\beta_{S}, d_{2}\right):=\left(\bar{p}_{*}(\beta), d_{2}(\beta)\right) \in W
$$

to denote the class of $\beta$ modulo extremal rays.

Given insertions

$$
A=\left(a_{1}, \ldots, a_{n}\right) \in H(\tilde{E})^{\oplus n}
$$

and a weighted partition

$$
(\varepsilon, \mu)=\left\{\left(\varepsilon_{1}, \mu_{1}\right), \ldots,\left(\varepsilon_{\rho}, \mu_{\rho}\right)\right\},
$$

the genus $g$ relative invariant $\langle A \mid \varepsilon, \mu\rangle_{g}$ is obtained by summing over classes $\beta=\beta_{Z}+d_{2} \gamma \in$ $\mathrm{NE}(\tilde{E})$ with

$$
\sum_{j=1}^{n} \operatorname{deg} a_{j}+\sum_{j=1}^{\rho} \operatorname{deg} \varepsilon_{j}=\left(c_{1}(\tilde{E}) \cdot \beta\right)+(\operatorname{dim} \tilde{E}-3)(1-g)+n+\rho-|\mu| .
$$

In this case, $d_{2}=(E \cdot \beta)=|\mu|$ is already fixed and non-negative.

Proposition 4.7. For an ordinary flop $\tilde{E} \rightarrow \tilde{E}^{\prime}$, to prove

$$
\mathscr{F}\langle A \mid \varepsilon, \mu\rangle_{g, \beta_{S}} \cong\langle\mathscr{F} A \mid \varepsilon, \mu\rangle_{g, \beta_{S}}
$$

for any $A \in H(\tilde{E})^{\oplus n}, \beta_{S} \in \mathrm{NE}(S)$ and $(\varepsilon, \mu)$ up to genus $g \leqslant g_{0}$, it is enough to prove the $\mathscr{F}$-invariance for descendent invariants of $f$-special type. Namely,

$$
\mathscr{F}\left\langle A, \tau_{k_{1}} \varepsilon_{1}, \ldots, \tau_{k_{\rho}} \varepsilon_{\rho}\right\rangle_{g, \beta_{S}, d_{2}}^{\tilde{E}} \cong\left\langle\mathscr{F} A, \tau_{k_{1}} \varepsilon_{1}, \ldots, \tau_{k_{\rho}} \varepsilon_{\rho}\right\rangle_{g, \beta_{S}, d_{2}}^{\tilde{E}^{\prime}}
$$




\section{INVARIANCE OF QUANTUM RINGS I}

for any $A \in H(\tilde{E})^{\oplus n}, k_{j} \in \mathbb{N} \cup\{0\}, \varepsilon_{j} \in H(E), \beta_{S} \in \mathrm{NE}(S)$ and $d_{2} \geqslant 0$ up to genus $g \leqslant g_{0}$.

Proof. The proof proceeds inductively on the 5-tuple

$$
\left(g, \beta_{S},|\mu|=d_{2}, n, \rho\right)
$$

in lexicographical order, with $\rho$ in the reverse order.

Given $\left\langle a_{1}, \ldots, a_{n} \mid \varepsilon, \mu\right\rangle_{g, \beta_{S}}$, since $\rho \leqslant|\mu|$, there are only finitely many 5-tuples of lower order. The proposition holds for those cases by the induction hypothesis.

We apply degeneration to the normal cone for $Z \hookrightarrow \tilde{E}$ to get $W \rightarrow \mathbb{A}^{1}$. Then $W_{0}=Y_{1} \cup Y_{2}$ with $\pi: Y_{1} \cong P\left(\mathscr{O}_{E}(-1,-1) \oplus \mathscr{O}\right) \rightarrow E$ a $P^{1}$ bundle and $Y_{2} \cong \tilde{E}$. Denote by $E_{0}=E=Y_{1} \cap Y_{2}$ and $E_{\infty} \cong E$ the zero and infinity divisors of $Y_{1}$, respectively.

The idea is to analyze the degeneration formula for

$$
\left\langle a_{1}, \ldots, a_{n}, \tau_{\mu_{1}-1} \varepsilon_{1}, \ldots, \tau_{\mu_{\rho}-1} \varepsilon_{\rho}\right\rangle_{g, \beta_{S}, d_{2}}^{\tilde{E}},
$$

since formally it is obtained by summing over the same curve classes $\beta$ as those in $\left\langle a_{1}, \ldots, a_{n}\right|$ $\varepsilon, \mu\rangle_{g, \beta_{S}}$ with

$$
\sum_{j=1}^{n} \operatorname{deg} a_{j}+|\mu|-\rho+\sum_{j=1}^{\rho}\left(\operatorname{deg} \varepsilon_{j}+1\right)=\left(c_{1}(\tilde{E}) \cdot \beta\right)+(\operatorname{dim} \tilde{E}-3)(1-g)+n+\rho .
$$

As in the proof of Proposition 4.3, we consider the generating series of invariants with possibly disconnected domain curves while keeping the total contact order $d_{2}=|\mu|$. Then we degenerate the series according to the contact order.

We first analyze the splitting of the curve classes. Under $N_{1}(\tilde{E})=i_{*} N_{1}(Z) \oplus \mathbb{Z} \gamma$, the class $\beta=\beta_{Z}+d_{2} \gamma$ may be split into

$$
\beta^{1} \in \mathrm{NE}\left(Y_{1}\right) \subset \mathrm{NE}(E) \oplus \mathbb{Z} \bar{\gamma}, \quad \beta^{2} \in \mathrm{NE}\left(Y_{2}\right) \equiv \mathrm{NE}(\tilde{E}),
$$

where

$$
\left(\beta^{1}, \beta^{2}\right)=\left(\beta_{E}^{1}+c \bar{\gamma}, \beta_{Z}^{2}+e \gamma\right)
$$

is subject to the condition $\phi_{*} \beta^{1}+p_{*} \beta^{2}=\beta$, that is,

$$
\bar{\phi}_{*} \beta_{E}^{1}+\beta_{Z}^{2}=\beta_{Z}, \quad c=d_{2} \geqslant 0,
$$

and to the contact order relation

$$
e=\left(E \cdot \beta^{2}\right)^{\tilde{E}}=\left(E \cdot \beta^{1}\right)^{Y_{1}}=c+\left(E \cdot \beta_{E}^{1}\right)^{Y_{1}}=d_{2}-\left(E \cdot \beta_{E}^{1}\right)^{\tilde{E}} .
$$

As an effective class in $E$, the class $\beta_{E}^{1}$ is also effective in $\tilde{E}$, hence $\beta_{E}^{1}=\zeta+m \gamma$ with $\zeta \in \mathrm{NE}(Z)$ and $m \in \mathbb{Z}$. It is clear that $\zeta=\bar{\phi}_{*} \beta_{E}^{1}$ and $m=\left(E \cdot \beta_{E}^{1}\right)^{\tilde{E}}$. It should be noticed that

$$
e=d_{2}-m
$$

is not necessarily less than $d_{2}$, since $m$ may be negative. This causes no trouble since we always have

$$
\beta-\beta^{2}=\left(\beta_{Z}+d_{2} \gamma\right)-\left(\beta_{Z}^{2}+e \gamma\right)=\bar{\phi}_{*} \beta_{E}^{1}+m \gamma=\beta_{E}^{1} \geqslant 0 .
$$

The equality holds if and only if $\beta_{E}^{1}=0$ and in that case we arrive at fiber class integrals on $\left(Y_{1}, E\right)$ with $\beta^{1}=d_{2} \bar{\gamma}$.

In fact, more is true. It is automatic that $[\beta]>\left[\beta^{2}\right]$ under the curve class splitting. The equality $[\beta]=\left[\beta^{2}\right]$ occurs if and only if $\beta_{E}^{1}$ consists of extremal rays $d_{1} \ell$. But extremal rays 
must stay inside $Z$, hence we again conclude that $\beta_{E}^{1}=0$ and get fiber integrals on $\left(Y_{1}, E\right)$. No summation over extremal rays is needed for these integrals.

Next, we analyze the splitting of the cohomology insertions.

It is sufficient to consider $\left(\varepsilon_{1}, \ldots, \varepsilon_{\rho}\right)=\mathbf{e}_{I}=\left(\mathbf{e}_{i_{1}}, \ldots, \mathbf{e}_{i_{\rho}}\right)$. Since $\left.\varepsilon_{i}\right|_{Z}=0$, one may choose the cohomology lifting $\varepsilon_{i}(0)=\left(\iota_{1 *} \varepsilon_{i}, 0\right)$. This ensures that insertions of the form $\tau_{k} \varepsilon$ go to the $Y_{1}$ side in the degeneration formula.

For a general cohomology insertion $\alpha \in H(\tilde{E})$, by Lemma 4.1, the lifting can be chosen to be $\alpha(0)=(a, \alpha)$ for some $a$. From $\alpha(0)=(a, \alpha)$ and $\mathscr{F} \alpha(0)=\left(a^{\prime}, \mathscr{F} \alpha\right)$, Lemma 4.2 implies $a=a^{\prime}$.

As before, the relative invariants on $\left(Y_{1}, E\right)$ can be regarded as constants under $\mathscr{F}$. Then

$$
\begin{aligned}
& \left\langle a_{1}, \ldots, a_{n}, \tau_{\mu_{1}-1} \mathbf{e}_{i_{1}}, \ldots, \tau_{\mu_{\rho}-1} \mathbf{e}_{i_{\rho}}\right\rangle_{g, \beta_{S}, d_{2}}^{\bullet \tilde{E}} \\
& \quad=\sum_{\mu^{\prime}} m\left(\mu^{\prime}\right) \sum_{I^{\prime}}\left\langle\tau_{\mu_{1}-1} \mathbf{e}_{i_{1}}, \ldots, \tau_{\mu_{\rho}-1} \mathbf{e}_{i_{\rho}} \mid \mathbf{e}^{I^{\prime}}, \mu^{\prime}\right\rangle_{0,0}^{\bullet\left(Y_{1}, E\right)}\left\langle a_{1}, \ldots, a_{n} \mid \mathbf{e}_{I^{\prime}}, \mu^{\prime}\right\rangle_{g, \beta_{S}}^{(\tilde{E}, E)}+R,
\end{aligned}
$$

where the main terms consist of the invariants in the sum whose factors labeled by $(\tilde{E}, E)$ admit the highest order with respect to the first four induction parameters

$$
\left(g, \beta_{S},|\mu|=d_{2}, n\right) .
$$

In fact, the potentially highest-order term $\left\langle a_{1}, \ldots, a_{n} \mid \mathbf{e}_{I}, \mu\right\rangle_{g, \beta_{S}}^{(\tilde{E}, E)}$ occurs, by the dimension count at the beginning of the proof. Yet it is not clear a priori whether it is also the highest-order term in $\rho$.

For the remaining terms, combined in $R$, a term is in $R$ if each connected component of its relative invariants on $(\tilde{E}, E)$ has lower inductive order with respect to the first four induction parameters. Notice that disconnected invariants on $(\tilde{E}, E)$ must lie in $R$.

For the main terms, by the genus constraint and the fact that the invariants on $(\tilde{E}, E)$ are connected, the invariants on $\left(Y_{1}, E\right)$ must be of genus zero and the connected components are indexed by the contact points. Also, each connected invariant contains fiber integrals with total fiber class $\beta^{1}=d_{2} \bar{\gamma}$.

To get constraints concerning $\left(\mathbf{e}_{I^{\prime}}, \mu^{\prime}\right)$ and $\rho^{\prime}$ on the main terms, we recall the dimension count on $\tilde{E}$ and $(\tilde{E}, E)$. Let $D=\left(c_{1}(\tilde{E}) \cdot \beta\right)+(\operatorname{dim} \tilde{E}-3)(1-g)$. For the absolute invariant on $\tilde{E}$, we have

$$
\sum_{j=1}^{n} \operatorname{deg} a_{j}+|\mu|-\rho+\sum_{j=1}^{\rho}\left(\operatorname{deg} \mathbf{e}_{i_{j}}+1\right)=D+n+\rho,
$$

while on $(\tilde{E}, E)$ (notice that now $\left.\left(c_{1}(\tilde{E}) \cdot \beta^{2}\right)=\left(c_{1}(\tilde{E}) \cdot \beta\right)\right)$, we have

$$
\sum_{j=1}^{n} \operatorname{deg} a_{j}+\sum_{j=1}^{\rho^{\prime}} \operatorname{deg} \mathbf{e}_{i_{j}^{\prime}}=D+n+\rho^{\prime}-\left|\mu^{\prime}\right| .
$$

Hence $\left(\mathbf{e}_{I}, \mu\right)$ occurs in the pairs $\left(\mathbf{e}_{I^{\prime}}, \mu^{\prime}\right)$ and, in particular, $R$ is $\mathscr{F}$-invariant by induction. Moreover,

$$
\operatorname{deg} \mathbf{e}_{I}-\operatorname{deg} \mathbf{e}_{I^{\prime}}=\rho-\rho^{\prime} .
$$

We will show that in the main terms, the highest-order term with respect to all five parameters, consists of the single term

$$
C(\mu)\left\langle a_{1}, \ldots, a_{n} \mid \mathbf{e}_{I}, \mu\right\rangle_{g, \beta_{S}}^{(\tilde{E}, E)}
$$




\section{INVARIANCE OF QUANTUM RINGS I}

with $C(\mu) \neq 0$.

For any $\left(\mathbf{e}_{I^{\prime}}, \mu^{\prime}\right)$ in the main terms, consider the splitting of weighted partitions

$$
\left(\mathbf{e}_{I}, \mu\right)=\coprod_{k=1}^{\rho^{\prime}}\left(\mathbf{e}_{I^{k}}, \mu^{k}\right)
$$

according to the connected components of the relative moduli of $\left(Y_{1}, E\right)$, which are indexed by the contact points of $\mu^{\prime}$.

Since relative invariants on $P^{1}$ bundles over $E$ along fiber classes can be computed by pairing cohomology classes in $E$ with certain Gromov-Witten invariants in the fiber $P^{1}$ (cf. [MP06, Section 1.2]), we must have $\operatorname{deg} \mathbf{e}_{I^{k}}+\operatorname{deg} \mathbf{e}^{i^{\prime}} \leqslant \operatorname{dim} E$ to get non-trivial invariants. That is,

$$
\operatorname{deg} \mathbf{e}_{I^{k}}=\sum_{j} \operatorname{deg} \mathbf{e}_{i_{j}^{k}} \leqslant \operatorname{dim} E-\operatorname{deg} \mathbf{e}^{i_{k}^{\prime}} \equiv \operatorname{deg} \mathbf{e}_{i_{k}^{\prime}}
$$

for each $k$. In particular, $\operatorname{deg} \mathbf{e}_{I} \leqslant \operatorname{deg} \mathbf{e}_{I^{\prime}}$; hence also $\rho \leqslant \rho^{\prime}$.

The case $\rho<\rho^{\prime}$ is handled by the induction hypothesis, so we assume $\rho=\rho^{\prime}$; then $\operatorname{deg} \mathbf{e}_{I^{k}}=$ $\operatorname{deg} \mathbf{e}_{i_{k}^{\prime}}$ for each $k=1, \ldots, \rho^{\prime}$. In particular, $I^{k} \neq \emptyset$ for each $k$. This implies that $I^{k}$ consists of a single element. By reordering we may assume $I^{k}=\left\{i_{k}\right\}$ and $\left(\mathbf{e}_{I^{k}}, \mu^{k}\right)=\left\{\left(\mathbf{e}_{i_{k}}, \mu_{k}\right)\right\}$.

Since the relative invariants on $Y_{1}$ contain genus zero fiber integrals, the virtual dimension for each $k$ (connected component of the relative virtual moduli) is

$$
2 \mu_{k}^{\prime}+\left(\operatorname{dim} Y_{1}-3\right)+1+\left(1-\mu_{k}^{\prime}\right)=\left(\mu_{k}-1\right)+\left(\operatorname{deg} \mathbf{e}_{i_{k}}+1\right)+\left(\operatorname{dim} E-\operatorname{deg} \mathbf{e}_{i_{k}^{\prime}}\right) .
$$

Together with $\operatorname{deg} \mathbf{e}_{i_{k}}=\operatorname{deg} \mathbf{e}_{i_{k}^{\prime}}$, this implies

$$
\mu_{k}^{\prime}=\mu_{k}, \quad k=1, \ldots, \rho .
$$

From the consideration of fiber class invariants and

$$
\operatorname{deg} \mathbf{e}_{i_{k}}+\operatorname{deg} \mathbf{e}^{i_{k}^{\prime}}=\operatorname{dim} E,
$$

$\mathbf{e}_{i_{k}}$ and $\mathbf{e}^{i_{k}^{\prime}}$ must be Poincaré dual to get a non-trivial integral over $E$. That is, $\mathbf{e}_{i_{k}^{\prime}}=\mathbf{e}_{i_{k}}$ for all $k$ and $\left(\mathbf{e}_{I^{\prime}}, \mu^{\prime}\right)=\left(\mathbf{e}_{I}, \mu\right)$. This gives the term we expect, where $C(\mu)$ is a product of non-trivial fiber class invariants

$$
\prod_{k=1}^{\rho}\left(\left\langle\tau_{\mu_{k}-1} \mathbf{e}_{i_{k}} \mid \mathbf{e}^{i_{k}}, \mu_{k}\right\rangle_{0, \mu_{k} \bar{\gamma}}^{\left(Y_{1}, E\right)} q^{\mu_{k} \bar{\gamma}}\right)=c_{\mu} q^{d_{2} \bar{\gamma}}
$$

with $c_{\mu} \neq 0$.

In order to compare this with the series $\left\langle a_{1}, \ldots, a_{n}, \tau_{\mu_{1}-1} \mathbf{e}_{i_{1}}, \ldots, \tau_{\mu_{\rho}-1} \mathbf{e}_{i_{\rho}}\right\rangle_{g, \beta_{S}, d_{2}}^{\tilde{E}}$, which satisfies the functional equation under $\mathscr{F}$ by assumption, we need only to match the formal variables involved. Under $\phi: Y_{1} \rightarrow \tilde{E}$ we set $q^{\bar{\gamma}} \mapsto q^{\gamma}$ and under $p: Y_{2} \cong \tilde{E} \rightarrow \tilde{E}$ we set $q^{\gamma} \mapsto q^{0}=1$. Similarly, we identify formal variables on the $\tilde{E}^{\prime}$ side. It is clear that these identifications commute with $\mathscr{F}$. Hence

$$
\mathscr{F}\left\langle a_{1}, \ldots, a_{n} \mid \mathbf{e}_{I}, \mu\right\rangle_{g, \beta_{S}}^{\tilde{E}} \cong\left\langle\mathscr{F} a_{1}, \ldots, \mathscr{F} a_{n} \mid \mathbf{e}_{I}, \mu\right\rangle_{g, \beta_{S}}^{\tilde{E}^{\prime}},
$$

and the proof of Proposition 4.7 is complete. 


\section{Y.-P. LeE, H.-W. Lin ANd C.-L. WANG}

\section{Reconstructions on local models}

In this section, $X$ and $X^{\prime}$ are the projective local models (double projective bundles over $S$ ) of the flop

$$
f: X=\tilde{E}=P_{Z}\left(N_{Z / X} \oplus \mathscr{O}\right) \rightarrow X^{\prime}=\tilde{E}^{\prime}=P_{Z^{\prime}}\left(N_{Z^{\prime} / X^{\prime}} \oplus \mathscr{O}\right) .
$$

Since we consider only genus zero invariants for the discussion on big quantum rings, the subscript for the genus will be omitted. One special feature for genus zero GW theory is that there exist several reconstruction theorems which allow us to deal with only some initial GW invariants.

By the Leray-Hirsch theorem,

$$
H(X)=H(S)[h, \xi] /\left(f_{F}(h), f_{N \oplus \mathscr{O}}(\xi)\right) .
$$

So every $a \in H(X)$ admits a canonical presentation $a=\bar{t} h^{i} \xi^{j}$ with $0 \leqslant i \leqslant r, 0 \leqslant j \leqslant r+1$ and $\bar{t} \in H(S)$. (In this case $\mathscr{F} a=\bar{t}(\mathscr{F} h)^{i}(\mathscr{F} \xi)^{j}=\bar{t}\left(\xi^{\prime}-h^{\prime}\right)^{i} \xi^{\prime j}$ for $i \leqslant r$ and for any $j$.) We abuse the notation by writing $\xi \mid a$ if $j \geqslant 1$.

Definition 5.1 ( $f$-special invariants). An insertion $\tau_{k} a$ is called special if $k \neq 0$ implies $\xi \mid a$. A (possibly) descendent invariant is $f$-special if it is not extremal (that is, $\left(\beta_{S}, d_{2}\right) \neq(0,0)$ ) and if all of its insertions are special. An $f$-special invariant is of type I if $\xi$ divides some insertion, otherwise it is of type II.

\subsection{Topological recursion relation and the divisor axiom}

ThEOREM 5.2. The $\mathscr{F}$-invariance for descendent invariants of $f$-special type is equivalent to the $\mathscr{F}$-invariance of big quantum rings.

Proof. We only need to prove " $\Leftarrow$ ".

Consider the generating series $\left\langle\tau_{k_{1}} a_{1}, \ldots, \tau_{k_{n}} a_{n}\right\rangle_{\beta_{S}, d_{2}}$ of $f$-special type with $\left(\beta_{S}, d_{2}\right) \neq(0,0)$. Let $k=\sum_{i} k_{i}$ be the total descendent degree. We will prove the theorem by induction on $k$.

If $k=0$, we may assume $n \geqslant 3$ by adding divisors $\xi$ or $D \in H^{2}(S)$ into the insertions. Since $(\xi \cdot \ell)=0=(D \cdot \ell)$, this only affects the series by a non-zero constant, hence the $\mathscr{F}$-invariance reduces to the case of the big quantum ring.

Now, let $k>0$. Without loss of generality, we assume $k_{1} \geqslant 1$. By induction the results hold for strictly smaller descendent degree and for any $n \geqslant 1$.

We first treat the case $n \geqslant 3$. By the topological recursion relation

$$
\psi_{1}=\left[D_{1 \mid 2,3}\right]^{\text {virt }}
$$

we get

$$
\left\langle\tau_{k_{1}} a_{1}, \ldots, \tau_{k_{n}} a_{n}\right\rangle_{\beta_{S}, d_{2}}=\sum_{\mu}\left\langle\tau_{k_{1}-1} a_{1}, \ldots, T_{\mu}\right\rangle_{\beta_{S}^{\prime}, d_{2}^{\prime}}\left\langle T^{\mu}, \tau_{k_{2}} a_{2}, \tau_{k_{3}} a_{3}, \ldots\right\rangle_{\beta_{S}^{\prime \prime}, d_{2}^{\prime \prime}}
$$

where the sum is over all splittings of curve classes such that $\left(\beta_{S}^{\prime}, d_{2}^{\prime}\right)+\left(\beta_{S}^{\prime \prime}, d_{2}^{\prime \prime}\right)=\left(\beta_{S}, d_{2}\right)$.

Notice that on the right-hand side, the case $\left(\beta_{S}^{\prime}, d_{2}^{\prime}\right)=(0,0)$ is excluded since $\xi \mid a_{1}$, so that case would lead to trivial invariants. The $\left(\beta_{S}^{\prime}, d_{2}^{\prime}\right)$ series is then $\mathscr{F}$-invariant since it has strictly smaller descendent order $k_{1}-1<k$. (Recall that on the $X^{\prime}$ side we may choose $\mathscr{F} T_{\mu}$ and $\mathscr{F} T^{\mu}$ for the splitting since $\mathscr{F}$ preserves the Poincaré pairing.)

The $\left(\beta_{S}^{\prime \prime}, d_{2}^{\prime \prime}\right)$ series is also $\mathscr{F}$-invariant: it has strictly smaller descendent degree and it has at least three insertions. So even if $\left(\beta_{S}^{\prime \prime}, d_{2}^{\prime \prime}\right)=(0,0)$, we still get the $\mathscr{F}$-invariance. 


\section{INVARIANCE OF QUANTUM RINGS I}

The case $n=1$ can be reduced to the case $n=2$ by the divisor equation for descendent invariants. Namely, let $b$ be either $\xi$ or a divisor coming from the base $S$ such that $b \cdot\left(\beta_{S}+d_{2} \gamma\right) \neq 0$. Then $(b \cdot \beta) \neq 0$ is independent of $d$ and

$$
\left\langle b, \tau_{k} a\right\rangle_{\beta_{S}, d_{2}}=(b \cdot \beta)\left\langle\tau_{k} a\right\rangle_{\beta_{S}, d_{2}}+\left\langle\tau_{k-1} a b\right\rangle_{\beta_{S}, d_{2}} .
$$

The case $n=2$ can be similarly reduced to the case $n=3$. If there is only one descendent insertion, say $\left\langle a_{1}, \tau_{k} a_{2}\right\rangle_{\beta_{S}, d_{2}}$, then

$$
\left\langle b, a_{1}, \tau_{k} a_{2}\right\rangle_{\beta_{S}, d_{2}}=(b \cdot \beta)\left\langle a_{1}, \tau_{k} a_{2}\right\rangle_{\beta_{S}, d_{2}}+\left\langle a_{1}, \tau_{k-1} a_{2} b\right\rangle_{\beta_{S}, d_{2}} .
$$

If there are two descendent insertions, say $\left\langle\tau_{l} a_{1}, \tau_{k-l} a_{2}\right\rangle_{\beta_{S}, d_{2}}$, then

$$
\left\langle b, \tau_{l} a_{1}, \tau_{k-l} a_{2}\right\rangle_{\beta_{S}, d_{2}}=(b \cdot \beta)\left\langle\tau_{l} a_{1}, \tau_{k-l} a_{2}\right\rangle_{\beta_{S}, d_{2}}+\left\langle\tau_{l-1} a_{1} b, \tau_{k-l} a_{2}\right\rangle_{\beta_{S}, d_{2}}+\left\langle\tau_{l} a_{1}, \tau_{k-l-1} a_{2} b\right\rangle_{\beta_{S}, d_{2}} .
$$

All the other series either are 3-point functions or have descendent degree that drops by 1 . Thus by induction the proof is complete.

\subsection{Divisorial reconstruction and quasi-linearity}

Theorem 5.2 completely reduces the analytic continuation problem to local models. However, in the actual determination of GW invariants (as we will see in later sections), another natural set of initial GW invariants consists of those with at most one descendent insertion. This suggests another reconstruction procedure.

DeFinition 5.3 (Quasi-linearity). We say that the flop $f$ is quasi-linear if for every special insertion $\alpha \in H(X) \cup \tau_{\bullet} H(E)$, every $\bar{t}_{i} \in H(S)$ and $\left(\beta_{S}, d_{2}\right) \neq(0,0)$, we have

$$
\mathscr{F}\left\langle\bar{t}_{1}, \ldots, \bar{t}_{n-1}, \alpha\right\rangle_{\beta_{S}, d_{2}}^{X} \cong\left\langle\bar{t}_{1}, \ldots, \bar{t}_{n-1}, \mathscr{F} \alpha\right\rangle_{\beta_{S}, d_{2}}^{X^{\prime}} .
$$

We call invariants with only one insertion not from the base elementary. Quasi-linearity is $\mathscr{F}$ invariance for elementary $f$-special invariants.

Notice that even for simple flops, the similar statement for descendent invariants is generally wrong if $\alpha=\tau_{k} a$ with $k>0$ but $a \notin H(E)$ (cf. [LLW10]).

Theorem 5.4. Suppose that $f$ is quasi-linear. Then all descendent invariants of $f$-special type are $\mathscr{F}$-invariant. Namely, for $\alpha=\left(\alpha_{1}, \ldots, \alpha_{n}\right)(n \geqslant 1)$ with $\alpha_{i} \in H(X) \cup \tau_{\bullet} H(E)$ and for $\left(\beta_{S}, d_{2}\right) \neq(0,0)$, we have

$$
\mathscr{F}\langle\alpha\rangle_{\beta_{S}, d_{2}}^{X} \cong\langle\mathscr{F} \alpha\rangle_{\beta_{S}, d_{2}}^{X^{\prime}}
$$

More precisely, any series of $f$-special type can be reconstructed, in an $\mathscr{F}$-compatible manner, from the extremal functions with $n \geqslant 3$ points and elementary $f$-special series.

We will prove the reconstruction by induction on $\left(\beta_{S}, d_{2}\right) \in W$, and then on $m$, which is the number of insertions not coming from base classes. This is based on the following observations:

(1) Under divisorial reconstruction, that is, $\psi_{i}+\psi_{j}=\left[D_{i \mid j}\right]^{\text {virt }}$, and for $L \in \operatorname{Pic}(X)$

$$
e_{i}^{*} L=e_{j}^{*} L+(\beta \cdot L) \psi_{j}-\sum_{\beta_{1}+\beta_{2}=\beta}\left(\beta_{1} \cdot L\right)\left[D_{i \beta_{1} \mid j \beta_{2}}\right]^{\mathrm{virt}}
$$

([LP04], cf. also [LLW10]), the degree $\beta$ is either preserved or split into effective classes $\beta=$ $\beta_{1}+\beta_{2}$. 
(2) When we sum over $\beta \in\left(d_{2} \gamma+\bar{\psi}^{*} \beta_{S} \cdot H_{r}+\mathbb{Z} \ell\right) \cap \mathrm{NE}(X)$, the splitting terms can usually be written as products of two generating series with no more marked points in a manner which will be clear in each context during the proof.

We also need to comment on the excluded case $\left(\beta_{S}, d_{2}\right)=(0,0)$.

(3) Let $\alpha_{i}=\tau_{k_{i}} a_{i}$. If $k=\sum k_{i} \neq 0$, say $\xi \mid a_{1}$, then the extremal invariants survive only for the case $\beta=0$. Since $\bar{M}_{0, n}(X, 0) \cong \bar{M}_{0, n} \times X$, we have

$$
\left\langle\tau_{k_{1}} a_{1}, \ldots, \tau_{k_{n}} a_{n}\right\rangle_{n, \beta=0}=\int_{\bar{M}_{0, n}} \psi_{1}^{k} \times \int_{X} a_{1} \cdots a_{n} .
$$

This is non-trivial only if $k=\operatorname{dim} \bar{M}_{0, n}=n-3$, and then

$$
\int_{X} a_{1} \cdots a_{n}=\int_{X^{\prime}} \mathscr{F} a_{1} \cdots \mathscr{F} a_{n}
$$

since the flop $f$ restricts to an isomorphism on $E$.

(4) For extremal invariants with $k=0$, since $\left.\xi\right|_{Z}=0$ and the extremal curves will always stay in $Z$, we get the trivial invariant if one of the insertions involves $\xi$. Hence by Theorem 3.9 the statement in the theorem still holds in this initial case except for the 2-point invariants $\left\langle\bar{t}_{1} h^{r}, \bar{t}_{2} h^{r}\right\rangle$. By the divisor axiom

$$
\delta_{h}\left\langle\bar{t}_{1} h^{r}, \bar{t}_{2} h^{r}\right\rangle=\left\langle h, \bar{t}_{1} h^{r}, \bar{t}_{2} h^{r}\right\rangle_{+},
$$

the 2-point invariants will satisfy the functional equation for $\mathscr{F}$-invariance up to analytic continuation only after they are incorporated with classical defect. Thus we may base our induction on $\left(\beta_{S}, d_{2}\right)=(0,0)$ with special care taken to handle this case.

Proof. Let $\left(\beta_{S}, d_{2}\right) \neq(0,0)$. If $m=1$, then we are done, so let $m \geqslant 2$.

Step 1. First we handle the type I case, that is, the case with the appearance of $\xi$ in some $\alpha_{i}$.

By reordering we may assume $\alpha_{n}=\tau_{s} \xi a$ for $s \geqslant 0$. Write

$$
\alpha_{1}=\bar{t}_{1} \tau_{k} h^{l} \xi^{j} \text {. }
$$

We will reduce $m$ by moving divisors from $\alpha_{1}$ into $\alpha_{n}$ in the order $\psi, h$ and $\xi$. This process is compatible with $\mathscr{F}$ since $\mathscr{F} a \cdot \mathscr{F} \xi=\mathscr{F}(a \cdot \xi)$.

For $\psi$, we use the equation

$$
\psi_{1}=-\psi_{n}+\left[D_{1 \mid n}\right]^{\mathrm{virt}}
$$

If $k \geqslant 1$, then $j \neq 0$ and we get

$$
\begin{aligned}
\left\langle\bar{t}_{1} \tau_{k} h^{l} \xi^{j}, \ldots, \tau_{s} \xi a\right\rangle_{\beta_{S}, d_{2}}= & -\left\langle\bar{t}_{1} \tau_{k-1} h^{l} \xi^{j}, \ldots, \tau_{s+1} \xi a\right\rangle_{\beta_{S}, d_{2}} \\
& +\sum_{\mu}\left\langle\bar{t}_{1} \tau_{k-1} h^{l} \xi^{j}, \ldots, T_{\mu}\right\rangle_{\beta_{S}^{\prime}, d_{2}^{\prime}}\left\langle T^{\mu}, \ldots, \tau_{s} \xi a\right\rangle_{\beta_{S}^{\prime \prime}, d_{2}^{\prime \prime}} .
\end{aligned}
$$

For each $i$, if one of $\left(\beta_{S}^{\prime}, d_{2}^{\prime}\right)$ and $\left(\beta_{S}^{\prime \prime}, d_{2}^{\prime \prime}\right)$ is $(0,0)$, then since both terms contain $\xi$, the splitting term must vanish. So we may assume

$$
\left(\beta_{S}^{\prime}, d_{2}^{\prime}\right)<\left(\beta_{S}, d_{2}\right) \text { and }\left(\beta_{S}^{\prime \prime}, d_{2}^{\prime \prime}\right)<\left(\beta_{S}, d_{2}\right),
$$

and these terms are done by the induction hypothesis. (By applying this procedure to $\alpha_{1}, \ldots$, $\alpha_{n-1}$ we may assume that the only descendent insertion is $\alpha_{n}$.) 


\section{INVARIANCE OF QUANTUM RINGS I}

For $h$, if $l \geqslant 1$, we use the divisor relation (5.1) for $L=h$ to get

$$
\begin{aligned}
\left\langle\bar{t}_{1} h^{l} \xi^{j}, \ldots, \tau_{s} \xi a\right\rangle_{\beta_{S}, d_{2}}= & \left\langle\bar{t}_{1} h^{l-1} \xi^{j}, \ldots, \tau_{s} \xi a h\right\rangle_{\beta_{S}, d_{2}}+\delta_{h}\left\langle\bar{t}_{1} h^{l-1} \xi^{j}, \ldots, \tau_{s+1} \xi a\right\rangle_{\beta_{S}, d_{2}} \\
& -\sum_{\mu} \delta_{h}\left\langle\bar{t}_{1} h^{l-1} \xi^{j}, \ldots, T_{\mu}\right\rangle_{\beta_{S}^{\prime}, d_{2}^{\prime}}\left\langle T^{\mu}, \ldots, \tau_{s} \xi a\right\rangle_{\beta_{S}^{\prime \prime}, d_{2}^{\prime \prime}}
\end{aligned}
$$

The only cases where the splitting term has one factor with the same $\left(\beta_{S}, d_{2}\right)$ and $m$ are of the form (denote by $\bar{t}_{*}$ some set of insertions $\alpha_{j} \in H(S)$ )

$$
\delta_{h}\left\langle\bar{t}_{1} h^{l-1} \xi^{j}, \bar{t}_{*}, T_{\mu}\right\rangle_{0,0}\left\langle T^{\mu}, \ldots, \tau_{s} \xi a\right\rangle_{\beta_{S}, d_{2}},
$$

where the first factor has $n^{\prime}$ points, or

$$
\delta_{h}\left\langle\bar{t}_{1} h^{l-1} \xi^{j}, \ldots, T_{\mu}\right\rangle_{\beta_{S}, d_{2}}\left\langle T^{\mu}, \bar{t}_{*}, \tau_{s} \xi a\right\rangle_{0,0} .
$$

But $l-1<r$ forces the invariants for the first factor of (5.4) to vanish: For $j \neq 0$ this is trivial. For $j=0$, the codimension is (cf. Section 3)

$$
\nu=|h|-\left(2 r+1+n^{\prime}-3\right)<2 r-2 r=0 .
$$

The invariants for the second factor of (5.5) also vanish since they contain $\xi$.

We notice that if $j=0$, the case $\left(\beta_{S}^{\prime}, d_{2}^{\prime}\right)=(0,0)$ may still support non-trivial invariants with three or more insertions which do not come from base classes. In that case $m$ decreases on the second factor of (5.4). For the other terms, the only possible appearance of type II invariants (that is, without $\xi$ insertion) is

$$
\delta_{h}\left\langle\bar{t}_{1} h^{l-1}, \ldots, T_{\mu}\right\rangle_{\beta_{S}^{\prime}, d_{2}^{\prime}}=\left\langle h, \bar{t}_{1} h^{l-1}, \ldots, T_{\mu}\right\rangle_{\beta_{S}^{\prime}, d_{2}^{\prime}},
$$

where $j=0$; this has at least three points and $(0,0)<\left(\beta_{S}^{\prime}, d_{2}^{\prime}\right)<\left(\beta_{S}, d_{2}\right)$.

For $\xi$, the argument is entirely similar. For $j \geqslant 1$, the divisor relation says that

$$
\begin{aligned}
\left\langle\bar{t}_{1} \xi^{j}, \ldots, \tau_{s} \xi a\right\rangle_{\beta_{S}, d_{2}}= & \left\langle\bar{t}_{1} \xi^{j-1}, \ldots, \tau_{s} \xi^{2} a\right\rangle_{\beta_{S}, d_{2}}+\delta_{\xi}\left\langle\bar{t}_{1} \xi^{j-1}, \ldots, \tau_{s+1} \xi a\right\rangle_{\beta_{S}, d_{2}} \\
& -\sum_{\mu} \delta_{\xi}\left\langle\bar{t}_{1} \xi^{j-1}, \ldots, T_{\mu}\right\rangle_{\beta_{S}^{\prime}, d_{2}^{\prime}}\left\langle T^{\mu}, \ldots, \tau_{s} \xi a\right\rangle_{\beta_{S}^{\prime \prime}, d_{2}^{\prime \prime}}
\end{aligned}
$$

We then have $\left(\beta_{S}^{\prime}, d_{2}^{\prime}\right)<\left(\beta_{S}, d_{2}\right)$ and $\left(\beta_{S}^{\prime \prime}, d_{2}^{\prime \prime}\right)<\left(\beta_{S}, d_{2}\right)$, as before. Notice that only type I invariants appear in the reduction.

Step 2. Next, we deal with the type II case: $\alpha_{i}=\bar{t}_{i} h^{l_{i}}$ for $1 \leqslant i \leqslant n$. In case $\beta_{S}=0$, we can add one $\xi$ into the insertions and then go back to Step 1. From (5.7), the pair $\left(\beta_{S}, d_{2}\right)$ will get smaller when the possible type II invariants appear again, so it is done by induction. Thus we can assume $\beta_{S} \neq 0$ here. By adding base divisors into the insertions we may always assume $n \geqslant 3$.

We cannot apply (5.1) to move divisors since it will produce non- $f$-special invariants. Instead, since $n \geqslant 3$ we may apply (3.1), the descendent-free form of the divisor relation, as we have used in the proof of Theorem 3.9.

Suppose $l_{1}>0$ and $l_{2}>0$ and move $h$ from $\alpha_{1}$ to $\alpha_{2}$. We run induction on $l_{1}$. Namely, we assume that the $\mathscr{F}$-invariant reduction holds for $\alpha_{1}=\bar{t}_{1} h^{j}$ with $j \leqslant l_{1}-1$. The initial case $j=0$ 
holds since $m$ drops by 1 . Then

$$
\begin{aligned}
\left\langle\bar{t}_{1} h^{l_{1}}, \bar{t}_{2} h^{l_{2}}, \alpha_{3}, \ldots\right\rangle_{\beta_{S}, d_{2}}= & \left\langle\bar{t}_{1} h^{l_{1}-1}, \bar{t}_{2} h^{l_{2}+1}, \alpha_{3}, \ldots\right\rangle_{\beta_{S}, d_{2}} \\
& +\sum_{\mu}\left\langle\bar{t}_{1} h^{l_{1}-1}, \alpha_{3}, \ldots, T_{\mu}\right\rangle_{\beta_{S}^{\prime}, d_{2}^{\prime}} \delta_{h}\left\langle\bar{t}_{2} h^{l_{2}}, \ldots, T^{\mu}\right\rangle_{\beta_{S}^{\prime \prime}, d_{2}^{\prime \prime}} \\
& -\delta_{h}\left\langle\bar{t}_{1} h^{l_{1}-1}, \ldots, T_{\mu}\right\rangle_{\beta_{S}^{\prime}, d_{2}^{\prime}}\left\langle\bar{t}_{2} h^{l_{2}}, \alpha_{3}, \ldots, T^{\mu}\right\rangle_{\beta_{S}^{\prime \prime}, d_{2}^{\prime \prime}}
\end{aligned}
$$

If $l_{2} \leqslant r-1$, the processes on $X$ and $X^{\prime}$ are clearly $\mathscr{F}$-compatible and the splitting terms are all handled by induction. Indeed, if $\left(\beta_{S}^{\prime}, d_{2}^{\prime}\right)=\left(\beta_{S}, d_{2}\right)$ and $m^{\prime}=m$, then $\left(\beta_{S}^{\prime \prime}, d_{2}^{\prime \prime}\right)=(0,0)$, which gives an extremal function with $m^{\prime \prime} \leqslant 2$. A codimension condition analogous to that in $(5.6)$ forces the term to vanish. A similar consideration applies to the case $\left(\beta_{S}^{\prime \prime}, d_{2}^{\prime \prime}\right)=\left(\beta_{S}, d_{2}\right)$ as well.

If $l_{2}=r$, the first term is no longer $\mathscr{F}$-compatible. The topological defect of the second insertion is given by Lemma 3.8: $\mathscr{F}\left(h^{r+1}\right)-(\mathscr{F} h)^{r+1}=(-1)^{r+1} \mathscr{F} \Theta_{r+1}$, where $\Theta_{r+1}$ is the dual class of pt $\cdot h^{r} \xi^{0}$. Meanwhile, the splitting terms also contain one term not of lower order in $\left(\beta_{S}, d_{2}\right)$ and $m$. By a codimension consideration analogous to that in (5.6), we have $T^{\mu}=\check{\bar{t}}_{2} h^{r}$, and the term is given by

$$
\left\langle\bar{t}_{1} h^{l_{1}-1}, \alpha_{3}, \ldots, \alpha_{n}, \bar{t}_{2} \Theta_{r+1}\right\rangle_{\beta_{S}, d_{2}} \delta_{h}\left\langle\bar{t}_{2} h^{r}, \check{\bar{t}}_{2} h^{r}\right\rangle_{0,0} .
$$

Comparing with its corresponding term on $X^{\prime}$

$$
\left\langle\bar{t}_{1} \mathscr{F} h^{l_{1}-1}, \mathscr{F} \alpha_{3}, \ldots, \mathscr{F} \alpha_{n}, \bar{t}_{2} \mathscr{F} \Theta_{r+1}\right\rangle_{\beta_{S}, d_{2}} \delta_{\mathscr{F} h}\left\langle\bar{t}_{2} \mathscr{F} h^{r}, \check{t}_{2} \mathscr{F} h^{r}\right\rangle_{0,0}
$$

and using the induction, we find the difference to be

$$
\begin{aligned}
& -\left\langle\bar{t}_{1} \mathscr{F} h^{l_{1}-1}, \mathscr{F} \alpha_{3}, \ldots, \mathscr{F} \alpha_{n}, \bar{t}_{2} \mathscr{F} \Theta_{r+1}\right\rangle_{\beta_{S}, d_{2}} \times(-1)^{r+1} \\
& \quad=-\left\langle\bar{t}_{1} \mathscr{F} h^{l_{1}-1}, \bar{t}_{2} \mathscr{F}\left(h^{r+1}\right), \ldots\right\rangle_{\beta_{S}, d_{2}}+\left\langle\bar{t}_{1} \mathscr{F} h^{l_{1}-1}, \bar{t}_{2}(\mathscr{F} h)^{r+1}, \ldots\right\rangle_{\beta_{S}, d_{2}} .
\end{aligned}
$$

This cancels the defect of the non- $\mathscr{F}$-compatible terms.

Thus the whole reduction is $\mathscr{F}$-invariant and the proof is complete.

\subsection{Witten-Dijkgraaf-Verlinde-Verlinde equations}

We may strengthen Theorem 5.4 to the following.

TheOREM 5.5. If quasi-linearity holds for elementary type I series

$$
\left\langle\bar{t}_{1}, \ldots, \bar{t}_{n-1}, \tau_{k} a \xi\right\rangle
$$

then $\mathscr{F}$-invariance holds for all series of $f$-special type.

The significance of this reduction will become clear after we introduce a practical method to calculate GW invariants. The proof is based on the following.

Proposition 5.6. Any type II series over $\left(\beta_{S}, d_{2}\right)$ can be transformed into a sum of products of (1) type I series over $\left(\beta_{S}^{\prime}, d_{2}^{\prime}\right) \leqslant\left(\beta_{S}, d_{2}\right)$, (2) type II series over $\beta_{S}^{\prime}<\beta_{S}$ and (3) extremal functions. Also, the processes can be done in an $\mathscr{F}$-compatible manner.

Indeed, with Proposition 5.6, Theorem 5.5 then follows from the proof of Theorem 5.4: simply replace step 2 by the proposition and run the induction. All type II special series eventually disappear. (Degenerate type II series with $\left(\beta_{S}, d_{2}\right)=(0,0)$ are simply extremal functions.)

The remainder of this subsection is devoted to the proof of Proposition 5.6. Notice that if $d_{2} \neq 0$, then this is trivial: by the divisor axiom,

$$
\left\langle a_{1}, \ldots, a_{n}\right\rangle_{\beta_{S}, d_{2}}=\left\langle a_{1}, \ldots, a_{n}, \xi\right\rangle_{\beta_{S}, d_{2}} / d_{2} .
$$




\section{INVARIANCE OF QUANTUM RINGS I}

Thus we consider $\left\langle a_{1}, \ldots, a_{n-1}, \bar{t}_{i} h^{j}\right\rangle_{\beta_{S}, 0}$ with $a_{1}, \ldots, a_{n-1} \in H(Z)$.

Let $\left\{\bar{T}_{i}\right\}$ be a basis for $H(S)$ and $\left\{\check{\bar{T}}_{i}\right\}$ its dual basis. We start with the case of 3-point functions $\left\langle a, b, \bar{T}_{i} h^{j}\right\rangle_{\beta_{S}, 0}$ for any $a, b \in H(Z)$. This certainly also includes the 1-point and 2-point cases by picking suitable $a, b \in H^{2}(S)$.

For any $c, d \in H(X)$, the WDVV equations

$$
\sum_{m, n} \partial_{i j m} F_{0} g^{m n} \partial_{n k l} F_{0}=\sum_{m, n} \partial_{i k m} F_{0} g^{m n} \partial_{n j l} F_{0}
$$

lead to the diagram

$$
[a \vee b \mapsto \xi c \vee \xi d]=[a \vee \xi c \mapsto b \vee \xi d]
$$

We apply it to split the curve classes over $\left(\beta_{S}, d_{2}=1\right)$ and get a linear equation

$$
\sum_{i, j}\left\langle a, b, \bar{T}_{i} h^{j}\right\rangle_{\beta_{S}, 0}\left\langle\check{\bar{T}}_{i} H_{r-j} \Theta_{r+1}, \xi c, \xi d\right\rangle_{0, d_{2}}=I_{c, d}
$$

where all terms on the left-hand sides of the WDVV equations with either (1) $\beta_{S}^{\prime}<\beta_{S}$, (2) $d_{2}^{\prime} \neq 0$ or $(3)$ basis class insertion $T_{\mu}=\bar{T}_{i} h^{j} \xi^{k}(k>0)$ from the diagonal splitting have been moved to the right-hand sides. Since the original right-hand sides of the WDVV equations are all type I series, any series in $I_{c, d}$ over $\left(\beta_{S}^{\prime}, d_{2}^{\prime}\right)$ must satisfy $\beta_{S}^{\prime}<\beta_{S}$ or $\left(\beta_{S}^{\prime}, d_{2}^{\prime}\right)=\left(\beta_{S}, 0\right)$.

Let $m=\sum_{i} h^{i}(S)$. We intend to form an $N \times N$ invertible system with $N=m(r+1)$. The virtual dimension of the second series is

$$
d_{2}(r+2)+2 r+1+s .
$$

Thus for $d_{2}=1$, we should require $|c|+|d|=r+\left|\bar{T}_{i}\right|+j$ to match the dimension.

Natural choices of $\{(c, d)\}$ are

$$
c=c_{k, l}:=\bar{T}_{k} \xi^{l}, \quad d=h^{r} .
$$

The set $\left\{c_{k, l}\right\}$ is partially ordered by $\left|\bar{T}_{k}\right|$ and then by $l$.

We claim that the resulting system is upper triangular with non-zero diagonal. Indeed,

$$
\left\langle\check{\bar{T}}_{i} H_{r-j} \Theta_{r+1}, \bar{T}_{k} \xi^{l+1}, \xi h^{r}\right\rangle_{0,1} \neq 0
$$

only if $\left|\bar{T}_{k}\right|+l=\left|\bar{T}_{i}\right|+j$.

The key point is to use the fiber bundle structure $\bar{M}_{0, n}(X, \beta) \rightarrow S$ for $\beta=d \ell+d_{2} \gamma$ as in the extremal case (where $d_{2}=0$ ). The fiber is given by the fiber bundle structure $\bar{M}_{0, n}$ of the toric local model for the simple flop case.

Thus if $\left|\bar{T}_{k}\right|>\left|\bar{T}_{i}\right|$, then $\left|\check{\bar{T}}_{i}\right|+\left|\bar{T}_{k}\right|>s$ and the invariant is zero. Even in the case $\left|\bar{T}_{k}\right|=\left|\bar{T}_{i}\right|$, and so $l=j$, we must have $\bar{T}_{k}=\bar{T}_{i}$ to avoid trivial invariants. The other cases $\left|\bar{T}_{k}\right|<\left|\bar{T}_{i}\right|$ belong to the strict upper triangular region which does not need our attention.

It remains to calculate the diagonal fiber series (the sum in taken over the possible nonnegative values of $d$ )

$$
\sum_{i}\left\langle\check{\bar{T}}_{i} H_{r-j} \Theta_{r+1}, \bar{T}_{i} \xi^{j+1}, \xi h^{r}\right\rangle_{0,1}=\left\langle h^{r-j}(\xi-h)^{r+1}, \xi^{j+1}, \xi h^{r}\right\rangle_{d_{2}=1}^{\text {simple }}
$$

We have done a similar calculation before for the extremal case in [LLW10, Proposition 3.8]. In the current case we have the following. 
Lemma 5.7. For simple flops, the fiber series in $d$ with $d_{2}=1$ are given by

$$
\left\langle h^{r-j}(\xi-h)^{r+1}, \xi^{j+1}, \xi h^{r}\right\rangle_{d_{2}=1}= \begin{cases}(-1)^{j} q^{\ell} q^{\gamma}, & 0 \leqslant j \leqslant r-1 \\ \left(1-(-1)^{r+1} q^{\ell}\right) q^{\gamma}, & j=r\end{cases}
$$

Proof. By applying the divisor relation to move one $\xi$ class with respect to $(i, j, k)=(2,1,3)$, we get (notice that $\xi(\xi-h)^{r+1}=0$ )

$$
\begin{aligned}
\left\langle h^{r-j}\right. & \left.(\xi-h)^{r+1}, \xi^{j+1}, \xi h^{r}\right\rangle_{d_{2}=1} \\
& =\sum_{\mu}\left\langle\xi^{j}, \xi h^{r}, T_{\mu}\right\rangle_{0} \delta_{\xi}\left\langle T^{\mu}, h^{r-j}(\xi-h)^{r+1}\right\rangle_{1}-\delta_{\xi}\left\langle\xi^{j}, T_{\mu}\right\rangle_{1}\left\langle T^{\mu}, h^{r-j}(\xi-h)^{r+1}, \xi h^{r}\right\rangle_{0} \\
& =\left\langle h^{r-j}(\xi-h)^{r+1}, \xi^{j+1} h^{r}\right\rangle_{1} .
\end{aligned}
$$

By another divisor relation (5.1), we can keep track of the 2-point invariants as follows:

$$
\begin{aligned}
\left\langle h^{r-j}(\xi-h)^{r+1}, \xi^{j+1} h^{r}\right\rangle_{1} & =\left\langle\psi h^{r-j}(\xi-h)^{r+1}, \xi^{j} h^{r}\right\rangle_{1}-\sum_{\mu} \delta_{\xi}\left\langle\xi^{j} h^{r}, T_{\mu}\right\rangle_{1}\left\langle T^{\mu}, h^{r-j}(\xi-h)^{r+1}\right\rangle_{0} \\
& =\left\langle\psi h^{r-j}(\xi-h)^{r+1}, \xi^{j} h^{r}\right\rangle_{1}=\cdots \\
& =\left\langle\psi^{j+1} h^{r-j}(\xi-h)^{r+1}, h^{r}\right\rangle_{1} .
\end{aligned}
$$

Here we use the fact that there is no extremal invariant with any insertion involving $\xi$ (notice that $(\xi-h)^{r+1}=\xi(\cdots)$ since $\left.h^{r+1}=0\right)$.

Next, we move the divisor classes $h$ in $h^{r}$ to the left one by one:

$$
\begin{aligned}
&\left\langle\psi^{j+1} h^{r-j}(\xi-h)^{r+1}, h^{r}\right\rangle_{1}=\left\langle\psi^{j+1} h^{r-j+1}(\xi-h)^{r+1}, h^{r-1}\right\rangle_{1}+\delta_{h}\left\langle\psi^{j+2} h^{r-j}(\xi-h)^{r+1}, h^{r-1}\right\rangle_{1} \quad-\sum_{\mu} \delta_{h}\left\langle h^{r-1}, T_{\mu}\right\rangle_{0}\left\langle T^{\mu}, \psi^{j+1} h^{r-j}(\xi-h)^{r+1}\right\rangle_{1} \\
&=\left\langle\psi^{j+1}(h+d \psi) h^{r-j}(\xi-h)^{r+1}, h^{r-1}\right\rangle_{1}=\cdots \\
&=\left\langle\psi^{j+1}(h+d \psi)^{r-1} h^{r-j}(\xi-h)^{r+1}, h\right\rangle_{1} .
\end{aligned}
$$

Note that $\left\langle h^{r-1}, T_{\mu}\right\rangle_{0}=0$ since the power of $h$ is less than $r$.

Finally, the divisor axiom helps us to obtain the result

$$
\begin{aligned}
& \left\langle\psi^{j+1}(h+d \psi)^{r-1} h^{r-j}(\xi-h)^{r+1}, h\right\rangle_{1} \\
& \quad=d\left\langle\psi^{j+1}(h+d \psi)^{r-1} h^{r-j}(\xi-h)^{r+1}\right\rangle_{1}+\left\langle h \psi^{j}(h+d \psi)^{r-1} h^{r-j}(\xi-h)^{r+1}\right\rangle_{1} \\
& \quad=\left\langle\psi^{j}(h+d \psi)^{r} h^{r-j}(\xi-h)^{r+1}\right\rangle_{1},
\end{aligned}
$$

which is the constant term in the $z$-expansion in

$$
\left\langle\sum_{k \geqslant 0} \frac{\psi^{k}}{z^{k}} z^{j}(h+d z)^{r} h^{r-j}(\xi-h)^{r+1}\right\rangle_{1}=z^{j+2} e_{1 *}\left(\frac{1}{z(z-\psi)} e_{1}^{*}(h+d z)^{r} h^{r-j}(\xi-h)^{r+1}\right) .
$$

According to the same discussion of quasi-linearity in [LLW10], if $d_{2}-d<0$, then $P_{\beta}$ vanishes after multiplication by $\xi$. Here $h^{r-j}(\xi-h)^{r+1}$ does contain at least one $\xi$. Hence we only need to consider $d_{2} \geqslant d$. Now $d_{2}=1$, thus $d=0$ or 1 .

If $d=0$, then $h^{r} h^{r-j}(\xi-h)^{r+1}$ is non-trivial only if $j=r$ and in this case we get $h^{r}(\xi-h)^{r+1}=$ $h^{r} \xi^{r+1}=$ pt. It is clear that the constant term of the $z$-expansion of

$$
z^{r+2} J_{\beta} \cdot \mathrm{pt}=z^{r+2} \frac{1}{(\xi-h+z)^{r+1}(\xi+z)} \cdot \mathrm{pt}
$$




\section{INVARIANCE OF QUANTUM RINGS I}

is equal to 1 .

If $d=1$, then $J_{\beta}=1 /(h+z)^{r+1}(\xi+z)$. Thus

$$
\begin{aligned}
z^{j+2} & \frac{(h+z)^{r} h^{r-j}(\xi-h)^{r+1}}{(h+z)^{r+1}(\xi+z)}=\frac{z^{j+2}}{z^{2}} \frac{h^{r-j}(\xi-h)^{r+1}}{(1+h / z)(1+\xi / z)} \\
= & z^{j} h^{r-j}(\xi-h)^{r+1}\left(1-\frac{h}{z}+\frac{h^{2}}{z^{2}}-\cdots(-1)^{j} \frac{h^{j}}{z^{j}}+\cdots\right)\left(1-\frac{\xi}{z}+\cdots\right) .
\end{aligned}
$$

Since $\xi(\xi-h)^{r+1}=0$, the constant term is given by

$$
(-1)^{j} h^{r}(\xi-h)^{r+1}=(-1)^{j} h^{r} \xi^{r+1}=(-1)^{j} .
$$

The proof is complete.

Now, we consider $n$-point functions with $n \geqslant 3$. The WDVV equation is for triple derivatives of the potential function with $g=0$. Let $t \in H^{>2}(X)$ be a general insertion without the fundamental class and divisors. Then we have

$$
\sum_{i, j}\left\langle a, b, \bar{T}_{i} h^{j}\right\rangle_{\beta_{S}, 0}(t)\left\langle\check{\bar{T}}_{i} H_{r-j} \Theta_{r+1}, \bar{T}_{k} \xi^{l+1}, \xi h^{r}\right\rangle_{0,1}(t)=I_{k, l}(t)
$$

where any series in $I_{c, d}$ over $\left(\beta_{S}^{\prime}, d_{2}^{\prime}\right)$ must satisfy $\beta_{S}^{\prime}<\beta_{S}$ or $\left(\beta_{S}^{\prime}, d_{2}^{\prime}\right)=\left(\beta_{S}, 0\right)$.

By dimension counting, one more marked point increases the virtual dimension by 1 , while $t$ has Chow degree more than 1 , so we find that

$$
\left\langle\check{\bar{T}}_{i} H_{r-j} \Theta_{r+1}, \bar{T}_{k} \xi^{l+1}, \xi h^{r}\right\rangle_{0,1}(t)=\left\langle\check{\bar{T}}_{i} H_{r-j} \Theta_{r+1}, \bar{T}_{k} \xi^{l+1}, \xi h^{r}\right\rangle_{0,1}
$$

is in fact independent of $t$ when $\left|\bar{T}_{i}\right|+j=\left|\bar{T}_{k}\right|+l$. The linear system (5.10) is thus $\mathscr{F}$-compatible by the quantum invariance of the simple flop case [LLW10].

In any case, if $\left|\bar{T}_{k}\right|>\left|\bar{T}_{i}\right|$, then the invariants are still zero. In particular, the $N \times N$ system is still upper triangular. Moreover, the diagonal entries are still given by the original 3-point (finite) series. Thus the series

$$
\left\langle a, b, \bar{T}_{i} h^{j}\right\rangle_{\beta_{S}, 0}(t)
$$

are solvable in terms of the expected terms.

\section{ACKNOWLEDGEMENTS}

The authors would like to thank the anonymous referee for pointing out several typographical errors in an earlier version of the paper.

\section{REFERENCES}

CK99 D. A. Cox and S. Katz, Mirror symmetry and algebraic geometry, Math. Surveys Monogr., vol. 68 (Amer. Math. Soc., Providence, RI, 1999); http://dx.doi.org/10.1090/surv/068.

FW08 B. Fu and C.-L. Wang, Motivic and quantum invariance under stratified Mukai flops, J. Differential Geom. 80 (2008), no. 2, 261-280; http://projecteuclid.org/euclid.jdg/ 1221066632.

ILLW12 Y. Iwao, Y.-P. Lee, H.-W. Lin and C.-L. Wang, Invariance of Gromov-Witten theory under a simple flop, J. reine angew. Math. 663 (2012), 67-90; http://dx. doi .org/10.1515/CRELLE. 2011.097. 
Kaw08 Y. Kawamata, Flops connect minimal models, Publ. Res. Inst. Math. Sci. 44 (2008), no. 2, 419-423; http://dx.doi.org/10.2977/prims/1210167332.

Li02 J. Li, A degeneration formula of $G W$-invariants, J. Differential Geom. 60 (2002), no. 2, 199293; http://projecteuclid.org/euclid.jdg/1090351102.

Lin10 H. W. Lin, Quantum invariance under $\mathbb{P}^{1}$ flops of type $(k+2, k)$, Algebraic Geometry in East Asia (Seoul, 2008), Adv. Stud. Pure Math., vol. 6 (Math. Soc. Japan, Tokyo, 2010), 271-300.

LLQW16 Y.-P. Lee, H.-W. Lin, F. Qu and C.-L. Wang, Invariance of quantum rings under ordinary flops III: A quantum splitting principle, Cambridge J. Math. 4 (2016), no. 3, 333-401; http: //dx.doi.org/10.4310/CJM.2016.v4.n3.a2.

LLW10 Y.-P Lee, H.-W. Lin and C.-L. Wang, Flops, motives, and invariance of quantum rings, Ann. of Math. 172 (2010), no. 1, 243-290; http://dx.doi.org/10.4007/annals.2010.172.243.

LLW12_, Analytic continuations of quantum cohomology, Fifth International Congress of Chinese Mathematicians. Part 1, 2, AMS/IP Stud. Adv. Math., vol. 51(1) (Amer. Math. Soc., Providence, RI, 2012), 269-282.

LLW16_ Invariance of quantum rings under ordinary flops II: A quantum Leray-Hirsch theorem, Algebr. Geom. 3, no. 5, (2016), 615-653, http://dx.doi.org/10.14231/AG-2016-027.

LP04 Y.-P. Lee and R. Pandharipande, A reconstruction theorem in quantum cohomology and quantum K-theory, Amer. J. Math. 126 (2004), no. 6, 1367-1379; http://dx.doi.org/10.1353/ ajm.2004.0049.

LR01 A.-M. Li and Y. Ruan, Symplectic surgery and Gromov-Witten invariants of CalabiYau 3-folds, Invent. Math. 145 (2001), no. 1, 151-218; http://dx.doi.org/10.1007/ s002220100146.

Man99 Yu. I. Manin, Frobenius manifolds, quantum cohomology, and moduli spaces, Amer. Math. Soc. Colloq. Publ., vol. 47 (Amer. Math. Soc., Providence, RI, 1999).

MP06 D. Maulik and R. Pandharipande, A topological view of Gromov-Witten theory, Topology 45 (2006), no. 5, 887-918; http://dx.doi.org/10.1016/j.top.2006.06.002.

Wan03 C.-L. Wang, K-equivalence in birational geometry and characterizations of complex elliptic genera, J. Algebraic Geom. 12 (2003), no. 2, 285-306; http://dx.doi.org/10.1090/ S1056-3911-02-00312-0.

Wan04_, K-equivalence in birational geometry, Second International Congress of Chinese Mathematicians, New Stud. Adv. Math., vol. 4 (Int. Press, Somerville, MA, 2004), 199-216.

Wan11 Q Quantum invariance under flop transitions, in Geometry and Analysis, No. 2, Adv. Lect. Math., vol. 18 (Int. Press, Somerville, MA, 2011), 347-370.

Wit93 E. Witten, Phases of $N=2$ theories in two dimensions, Nuclear Phys. B 403 (1993), no. 1-2, 159-222; http://dx.doi.org/10.1016/0550-3213(93)90033-L.

Yuan-Pin Lee yplee@math.utah.edu

Department of Mathematics, University of Utah, Salt Lake City, Utah 84112-0090, USA

Hui-Wen Lin linhw@math.ntu.edu.tw

Department of Mathematics and Taida Institute of Mathematical Sciences (TIMS), National Taiwan University, Taipei 10617, Taiwan

Chin-Lung Wang dragon@math.ntu.edu.tw

Department of Mathematics, Center for Advanced Studies in Theoretical Sciences (CASTS)

and

Taida Institute of Mathematical Sciences (TIMS), National Taiwan University, Taipei 10617, Taiwan 